Proc. Indian Acad. Sci. (Math. Sci.) Vol. 117, No. 1, February 2003, pp. 109-145.

Printed in India

\title{
Nonconforming h-p spectral element methods for elliptic problems
}

\author{
P K DUTT* ${ }^{*}$ N KISHORE KUMAR* and C S UPADHYAY ${ }^{\dagger}$ \\ ${ }^{*}$ Department of Mathematics; ${ }^{\dagger}$ Department of Aerospace Engineering, Indian Institute \\ of Technology Kanpur, Kanpur 208 016, India
}

MS received 20 February 2006; revised 23 July 2006

\begin{abstract}
In this paper we show that we can use a modified version of the h-p spectral element method proposed in [6/7|13|14] to solve elliptic problems with general boundary conditions to exponential accuracy on polygonal domains using nonconforming spectral element functions. A geometrical mesh is used in a neighbourhood of the corners. With this mesh we seek a solution which minimizes the sum of a weighted squared norm of the residuals in the partial differential equation and the squared norm of the residuals in the boundary conditions in fractional Sobolev spaces and enforce continuity by adding a term which measures the jump in the function and its derivatives at inter-element boundaries, in fractional Sobolev norms, to the functional being minimized. In the neighbourhood of the corners, modified polar coordinates are used and a global coordinate system elsewhere. A stability estimate is derived for the functional which is minimized based on the regularity estimate in [2]. We examine how to parallelize the method and show that the set of common boundary values consists of the values of the function at the corners of the polygonal domain. The method is faster than that proposed in [6]7[14] and the h-p finite element method and stronger error estimates are obtained.
\end{abstract}

Keywords. Geometrical mesh; stability estimate; least-squares solution; precondi- tioners; condition numbers; exponential accuracy.

\section{Introduction}

In [6]7|13 14] h-p spectral element methods for solving elliptic boundary value problems on polygonal domains using parallel computers were proposed. For problems with Dirichlet boundary conditions the spectral element functions were nonconforming. For problems with Neumann and mixed boundary conditions the spectral element functions had to be continuous at the vertices of the elements only. In this paper we propose a modified version of this method using nonconforming spectral element functions which works for general boundary conditions.

For simplicity of exposition we restrict ourselves to scalar problems although the method applies to elliptic systems too.

A method for obtaining a numerical solution to exponential accuracy for elliptic problems with analytic coefficients posed on a curvilinear polygon whose boundary is piecewise analytic with mixed Neumann and Dirichlet boundary conditions was first proposed by Babuska and Guo [3] within the framework of the finite element method. They were able to resolve the singularities which arise at the corners by using a geometrical mesh. This problem has also been examined by Karniadakis and Spencer in [11]. 
We also use a geometrical mesh to solve the same class of problems to exponential accuracy using h-p spectral element methods. In a neighbourhood of the corners modified polar coordinates $\left(\tau_{k}, \theta_{k}\right)$ are used, where $\tau_{k}=\ln r_{k}$ and $\left(r_{k}, \theta_{k}\right)$ are polar coordinates with the origin at the vertex $A_{k}$. Away from sectoral neighbourhoods of the corners a global coordinate system is used consisting of $\left(x_{1}, x_{2}\right)$ coordinates.

We now seek a solution which minimizes the sum of the squares of a weighted squared norm of the residuals in the partial differential equation and the sum of the squares of the residuals in the boundary conditions in fractional Sobolev norms and enforce continuity by adding a term which measures the sum of the squares of the jump in the function and its derivatives in fractional Sobolev norms to the functional being minimized. These computations are done using modified polar coordinates in sectoral neighbourhoods of the corners and a global coordinate system elsewhere in the domain. The spectral element functions are nonconforming. For the modified version of the h-p spectral element method examined here a stability estimate is proved which is based on the regularity estimate of Babuska and Guo in [2]. The proof is much simpler than that of the stability estimate in [67]. Moreover the error estimates are stronger.

The set of common boundary values for the numerical scheme consists of the values of the function at the vertices of the polygonal domain. Since the cardinality of the set of common boundary values is so small we can compute a nearly exact approximation to the Schur complement. Let $M$ denote the number of corner layers and $W$ denote the number of degrees of freedom in each independent variable of the spectral element functions, which are a tensor product of polynomials, and let $W$ be proportional to $M$. Then the method is faster than the h-p spectral element method in [6/7]14] by a factor of $O\left(W^{1 / 2}\right)$ and faster than the h-p finite element method by a factor of $O(W)$.

We now outline the contents of this paper. In $\S 2$ function spaces are defined and differentiability estimates are obtained. In $\S 3$ we state and prove stability estimates. In $\S 4$ the numerical scheme, which is based on these estimates, is described and in $\S 5$ error estimates are obtained. In $\S 6$ we examine the issues of parallelization and preconditioning. Finally $\S 7$ contains technical results which are needed to prove the stability theorem.

\section{Function spaces and differentiability estimates}

Let $\Omega$ be a curvilinear polygon with vertices $A_{1}, A_{2}, \ldots, A_{p}$ and corresponding sides $\Gamma_{1}, \Gamma_{2}, \ldots, \Gamma_{p}$ where $\Gamma_{i}$ joins the points $A_{i-1}$ and $A_{i}$. We shall assume that the sides $\bar{\Gamma}_{i}$ are analytic arcs, i.e.

$$
\bar{\Gamma}_{i}=\left\{\left(\varphi_{i}(\xi), \psi_{i}(\xi)\right) \mid \xi \in \bar{I}=[-1,1]\right\}
$$

with $\varphi_{i}(\xi)$ and $\psi_{i}(\xi)$ being analytic functions on $\bar{I}$ and $\left|\varphi_{i}^{\prime}(\xi)\right|^{2}+\left|\psi_{i}^{\prime}(\xi)\right|^{2} \geq \alpha>0$. By $\Gamma_{i}$ we mean the open arc, i.e. the image of $I=(-1,1)$.

Let the angle subtended at $A_{j}$ be $\omega_{j}$. We shall denote the boundary $\partial \Omega$ of $\Omega$ by $\Gamma$. Further, let $\Gamma=\Gamma^{[0]} \cup \Gamma^{[1]}, \Gamma^{[0]}=\bigcup_{i \in \mathscr{D}} \bar{\Gamma}_{i}, \Gamma^{[1]}=\bigcup_{i \in \mathscr{N}} \bar{\Gamma}_{i}$ where $\mathscr{D}$ is a subset of the set $\{i \mid i=1, \ldots, p\}$ and $\mathscr{N}=\{i \mid i=1, \ldots, p\} \backslash \mathscr{D}$. Let $x$ denote the vector $x=\left(x_{1}, x_{2}\right)$.

Let $\mathscr{L}$ be a strongly elliptic operator

$$
\mathscr{L}(u)=-\sum_{r, s=1}^{2}\left(a_{r, s}(x) u_{x_{s}}\right)_{x_{r}}+\sum_{r=1}^{2} b_{r}(x) u_{x_{r}}+c(x) u,
$$


where $a_{s, r}(x)=a_{r, s}(x), b_{r}(x), c(x)$ are analytic functions on $\bar{\Omega}$ and for any $\xi_{1}, \xi_{2} \in \mathbb{R}$ and any $x \in \bar{\Omega}$,

$$
\sum_{r, s=1}^{2} a_{r, s} \xi_{r} \xi_{s} \geq \mu_{0}\left(\xi_{1}^{2}+\xi_{2}^{2}\right)
$$

with $\mu_{0}>0$. In this paper we shall consider the boundary value problem

$$
\begin{gathered}
\mathscr{L} u=f \quad \text { on } \Omega \\
u=g^{[0]} \quad \text { on } \Gamma^{[0]}, \\
\left(\frac{\partial u}{\partial N}\right)_{A}=g^{[1]} \text { on } \Gamma^{[1]},
\end{gathered}
$$

where $(\partial u / \partial N)_{A}$ denotes the usual conormal derivative which we shall now define. Let $A$ denote the $2 \times 2$ matrix whose entries are given by

$$
A_{r, s}(x)=a_{r, s}(x)
$$

for $r, s=1,2$. Let $N=\left(N_{1}, N_{2}\right)$ denote the outward normal to the curve $\Gamma_{i}$ for $i \in \mathscr{N}$. Then $\left(\frac{\partial u}{\partial N}\right)_{A}$ is defined as follows:

$$
\left(\frac{\partial u}{\partial N}\right)_{A}(x)=\sum_{r, s=1}^{2} N_{r} a_{r, s} \frac{\partial u}{\partial x_{s}} .
$$

Moreover let the bilinear form induced by the operator $\mathscr{L}$ satisfy the inf-sup conditions. It shall be assumed that the given data $f$ is analytic on $\bar{\Omega}$ and $g^{[l]}, l=0,1$ is analytic on every closed $\operatorname{arc} \bar{\Gamma}_{i}$ and $g^{[0]}$ is continuous on $\Gamma^{[0]}$.

By $H^{m}(\Omega)$ we denote the Sobolev space of functions with square integrable derivatives of order $\leq m$ on $\Omega$ furnished with the norm

$$
\|u\|_{H^{m}(\Omega)}^{2}=\sum_{|\alpha| \leq m}\left\|D^{\alpha} u\right\|_{L^{2}(\Omega)}^{2} .
$$

Define $r_{i}(x)$ to be the Euclidean distance between $x$ and the vertex $A_{i}$ of $\Omega$. Let $\beta=$ $\left(\beta_{1}, \beta_{2}, \ldots, \beta_{p}\right)$ denote a $p$-tuple of real numbers, $0<\beta_{i}<1, i=1, \ldots, p$. For any integer $k$, let $\beta+k=\left(\beta_{1}+k, \beta_{2}+k, \ldots, \beta_{p}+k\right)$. Further, we denote

$$
\Phi_{\beta}(x)=\prod_{i=1}^{p} r_{i}^{\beta_{i}} \quad \text { and } \quad \Phi_{\beta+k}(x)=\prod_{i=1}^{p} r_{i}^{\beta_{i}+k} \text {. }
$$

Let $H_{\beta}^{m, l}(\Omega), m \geq l \geq 0, l$ an integer, denote the completion of the set of all infinitely differentiable functions under the norm

$$
\begin{aligned}
\|u\|_{H_{\beta}^{m, l}(\Omega)}^{2} & =\|u\|_{H^{l-1}(\Omega)}^{2}+\sum_{|\alpha|=k, k=l}^{m}\left\|D^{\alpha} u \Phi_{\beta+k-l}\right\|_{L^{2}(\Omega)}^{2}, \quad l \geq 1 \\
\|u\|_{H_{\beta}^{m, 0}(\Omega)}^{2} & =\sum_{|\alpha|=k, k=0}^{m}\left\|D^{\alpha} u \Phi_{\beta+k-l}\right\|_{L^{2}(\Omega)}^{2}, \quad l=0 .
\end{aligned}
$$

For $m=l=0$ we shall write $H_{\beta}^{0,0}(\Omega)=L_{\beta}(\Omega)$. 
Let $\gamma$ be part of the boundary $\Gamma$ of $\Omega$. Define $H_{\beta}^{m-\frac{1}{2}, l-\frac{1}{2}}(\gamma), m \geq l, l \geq 0$ to be the set of all functions $\phi$ on $\gamma$ such that there exists $f \in H_{\beta}^{m, l}(\Omega)$ with $\phi=\left.f\right|_{\gamma}$ and

$$
\|\phi\|_{H_{\beta}^{m-\frac{1}{2}, l-\frac{1}{2}}(\gamma)}=\inf _{f \in H_{\beta}^{m, l}(\Omega)}\left\{\|f\|_{H_{\beta}^{m, l}(\Omega)}\right\} .
$$

For $l$ an integer $0 \leq l \leq 2$, let

$$
\psi_{\beta}^{l}(\Omega)=\left\{u(x) \mid u \in H_{\beta}^{m, l}(\Omega), m \geq l\right\}
$$

and

$$
\begin{aligned}
\mathfrak{B}_{\beta}^{l}(\Omega)= & \left\{u ( x ) \left|u \in \psi_{\beta}^{l}(\Omega),\left\|\left|D^{\alpha} u\right| \Phi_{\beta+k-l}\right\|_{L^{2}(\Omega)} \leq C d^{k-l}(k-l) !\right.\right. \\
& \text { for }|\alpha|=k=l, l+1, \ldots ; d \geq 1, C \text { independent of } k\} .
\end{aligned}
$$

Let $Q \subseteq \mathbb{R}^{2}$ be an open set with a piecewise analytic boundary $\partial Q$ and $\gamma$ be part or whole of the boundary $\partial Q$. Finally $\mathfrak{B}_{\beta}^{l-\frac{1}{2}}(\gamma), 0 \leq l \leq 2$, denotes the space of all functions $\varphi$ for which there exists $f \in \mathfrak{B}_{\beta}^{l}(Q)$ such that $f=\varphi$ on $\gamma$.

Next as in [3] we introduce the space $\mathfrak{C}_{\beta}^{2}$ :

$$
\begin{aligned}
\mathfrak{C}_{\beta}^{2}(\Omega)= & \left\{u \in H_{\beta}^{2,2}(\Omega)|| D^{\alpha} u(x) \mid \leq C d^{k} k !\left(\Phi_{k+\beta-1}(x)\right)^{-1}\right. \\
& |\alpha|=k=1,2, \ldots ; C \geq 1 ; d \geq 1 \text { independent of } k\}
\end{aligned}
$$

The relationship between $\mathfrak{C}_{\beta}^{2}$ and $\mathfrak{B}_{\beta}^{2}$ is given by Theorem 2.2 of [3] which can be stated as follows:

$$
\mathfrak{B}_{\beta}^{2}(\Omega) \subseteq \mathfrak{C}_{\beta}^{2}(\Omega) .
$$

We need to state our regularity estimates in terms of local variables which are defined on a geometrical mesh imposed on $\Omega$ as in $\S 5$ of [3]. $\Omega$ is first divided into subdomains. Thus we divide $\Omega$ into $p$ subdomains $S^{1}, \ldots, S^{p}$, where $S^{i}$ denotes a domain which contains the vertex $A_{i}$ and no other, and on each $S^{i}$ we define a geometrical mesh. Let $\mathfrak{S}^{k}=\left\{\Omega_{i, j}^{k}, j=\right.$ $\left.1, \ldots, J_{k}, i=1, \ldots, I_{k, j}\right\}$ be a partition of $S^{k}$ and let $\mathfrak{S}=\bigcup_{k=1}^{p} \mathfrak{S}^{k}$. The geometrical mesh imposed on $\Omega$ is as shown in figure 1 .

We now put some restrictions on $\mathfrak{S}$. Let $\left(r_{k}, \theta_{k}\right)$ denote polar coordinates with center at $A_{k}$. Let $\tau_{k}=\ln r_{k}$. Choose $\rho$ so that the curvilinear sector $\Omega^{k}$ with sides $\Gamma_{k}$ and $\Gamma_{k+1}$ bounded by the circular arc $B_{\rho}^{k}$, center at $A_{k}$ and radius $\rho$ satisfies

$$
\Omega^{k} \subseteq \bigcup_{\Omega_{i, j}^{k} \in \mathfrak{S}^{k}} \bar{\Omega}_{i, j}^{k}
$$

$\Omega^{k}$ may be represented as

$$
\Omega^{k}=\left\{\left(x_{1}, x_{2}\right) \in \Omega: 0<r_{k}<\rho\right\} .
$$




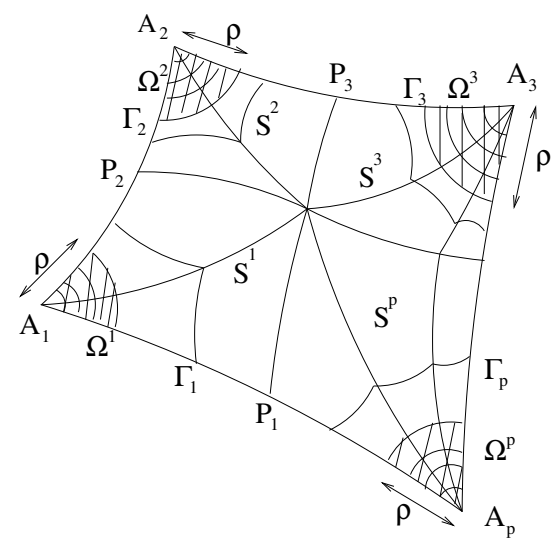

Figure 1. Geometrical mesh with $M$ layers in the radial direction.

Let $\gamma_{i, j, l}^{k}, 1 \leq l \leq 4$ be the side of the quadrilateral $\Omega_{i, j}^{k} \in \mathfrak{S}$. Then it is assumed that

$$
\begin{aligned}
& \gamma_{i, j, l}^{k}:\left\{\begin{array}{l}
x_{1}=h_{i, j}^{k} \varphi_{i, j, l}^{k}(\xi), \\
x_{2}=h_{i, j}^{k} \psi_{i, j, l}^{k}(\xi),
\end{array}\right. \\
& \gamma_{i, j, l}^{k}:\left\{\begin{array}{l}
x_{1}=h_{i, j}^{k} \varphi_{i, j, l}^{k}(\eta), \quad-1 \leq \eta \leq 1, \quad l=2,4 \\
x_{2}=h_{i, j}^{k} \psi_{i, j, l}^{k}(\eta),
\end{array}\right.
\end{aligned}
$$

and that for some $C \geq 1$ and $L \geq 1$ independent of $i, j, k$ and $l$,

$$
\left|\frac{d^{t}}{d s^{t}} \varphi_{i, j, l}^{k}(s)\right|,\left|\frac{d^{t}}{d s^{t}} \psi_{i, j, l}^{k}(s)\right| \leq C L^{t} t !, \quad t=1,2, \ldots
$$

We shall place further restrictions on the geometric mesh imposed on $\Omega^{k}$ later. Some of the elements may be curvilinear triangles.

Let $\left(r_{k}, \theta_{k}\right)$ be polar coordinates with center at $A_{k}$. Then $\Omega^{k}$ is the open set bounded by the curvilinear arcs $\Gamma_{k}, \Gamma_{k+1}$ and a portion of the circle $r_{k}=\rho$. We divide $\Omega^{k}$ into

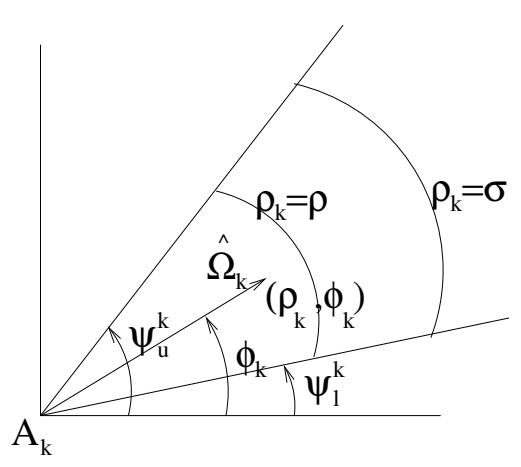

Figure 2. Curvilinear sectors.

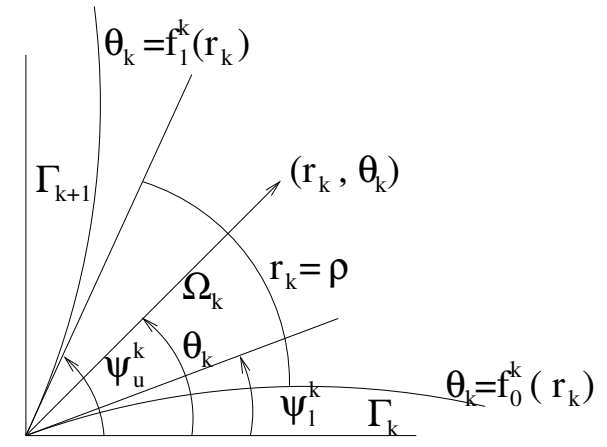

$\mathrm{A}_{\mathrm{k}}$ 
curvilinear rectangles by drawing $M$ circular arcs $r_{k}=\sigma_{j}^{k}=\rho \mu_{k}^{M+1-j}, j=2, \ldots, M+1$, where $\mu_{k}<1$ and $I_{k}-1$ analytic curves $C_{2}, \ldots, C_{I_{k}}$ whose exact form shall be prescribed in what follows. Let $\sigma_{1}^{k}=0$. Thus $I_{k, j}=I_{k}$ for $j \leq M$; in fact, we shall let $I_{k, j}=I_{k}$ for $j \leq M+1$. Moreover $I_{k, j} \leq I$ for all $k, j$ where $I$ is a fixed constant. Let

$$
\Gamma_{k+j}=\left\{\left(r_{k}, \theta_{k}\right) \mid \theta_{k}=f_{j}^{k}\left(r_{k}\right), \quad 0<r_{k}<\rho\right\},
$$

$j=0,1$ in a neighbourhood of $A_{k}$ in $\Omega^{k}$. Then the mapping

$$
r_{k}=\rho_{k}, \theta_{k}=\frac{1}{\left(\psi_{u}^{k}-\psi_{l}^{k}\right)}\left[\left(\phi_{k}-\psi_{l}^{k}\right) f_{1}^{k}\left(\rho_{k}\right)-\left(\phi_{k}-\psi_{u}^{k}\right) f_{0}^{k}\left(\rho_{k}\right)\right],
$$

where $f_{j}^{k}$ is analytic in $\rho_{k}$ for $j=0,1$, maps locally the cone

$$
\left\{\left(\rho_{k}, \phi_{k}\right): 0<\rho_{k}<\sigma, \psi_{l}^{k}<\phi_{k}<\psi_{u}^{k}\right\}
$$

onto a set containing $\Omega^{k}$ as in $\S 3$ of [3]. The functions $f_{j}^{k}$ satisfy $f_{0}^{k}(0)=\psi_{l}^{k}, f_{1}^{k}(0)=\psi_{u}^{k}$ and $\left(f_{j}^{k}\right)^{\prime}(0)=0$ for $j=0,1$. It is easy to see that the mapping defined in (2.8) has two bounded derivatives in a neighbourhood of the origin which contains the closure of the open set

$$
\hat{\Omega}^{k}=\left\{\left(\rho_{k}, \phi_{k}\right): 0<\rho_{k}<\rho, \psi_{l}^{k}<\phi_{k}<\psi_{u}^{k}\right\} .
$$

We choose the $I_{k}-1$ curves $C_{2}, \ldots, C_{I_{k}}$ as

$$
C_{i}: \phi_{k}\left(r_{k}, \theta_{k}\right)=\psi_{i}^{k}
$$

for $i=2, \ldots, I_{k}$. Here

$$
\psi_{l}^{k}=\psi_{1}^{k}<\psi_{2}^{k}<\cdots<\psi_{I_{k}+1}^{k}=\psi_{u}^{k} .
$$

Let $\Delta \psi_{i}^{k}=\psi_{i+1}^{k}-\psi_{i}^{k}$. Then $\left\{\psi_{i}^{k}\right\}_{i, k}$ are chosen so that

$$
\max _{i, k}\left(\Delta \psi_{i}^{k}\right)<\lambda\left(\min _{i, k}\left(\Delta \psi_{i}^{k}\right)\right)
$$

for some constant $\lambda$. Another set of local variables $\left(\tau_{k}, \theta_{k}\right)$ is needed in a neighbourhood of $\Omega^{k}$ where

$$
\tau_{k}=\ln r_{k} .
$$

In addition, we need one final set of local variables $\left(v_{k}, \phi_{k}\right)$ in the cone

$$
\left\{\left(\rho_{k}, \phi_{k}\right): 0 \leq \rho_{k} \leq \rho, \psi_{l}^{k} \leq \phi_{k} \leq \psi_{u}^{k}\right\},
$$

where

$$
v_{k}=\ln \rho_{k} .
$$

Let $S_{\mu}^{k}=\left\{\left(r_{k}, \theta_{k}\right): 0 \leq r_{k} \leq \mu\right\} \cap \Omega$. Then the image $\hat{S}_{\mu}^{k}$ in $\left(v_{k}, \phi_{k}\right)$ variables of $S_{\mu}^{k}$ is given by

$$
\hat{S}_{\mu}^{k}=\left\{\left(v_{k}, \phi_{k}\right):-\infty \leq v_{k} \leq \ln \mu, \psi_{l}^{k} \leq \phi_{k} \leq \psi_{u}^{k}\right\} .
$$


Now the relationship between the variables $\left(\tau_{k}, \theta_{k}\right)$ and $\left(v_{k}, \phi_{k}\right)$ is given by $\left(\tau_{k}, \theta_{k}\right)=$ $M^{k}\left(v_{k}, \phi_{k}\right)$, viz.

$$
\begin{aligned}
\tau_{k} & =v_{k}, \\
\theta_{k} & =\frac{1}{\left(\psi_{u}^{k}-\psi_{l}^{k}\right)}\left[\left(\phi_{k}-\psi_{l}^{k}\right) f_{1}^{k}\left(\mathrm{e}^{v_{k}}\right)-\left(\phi_{k}-\psi_{u}^{k}\right) f_{0}^{k}\left(\mathrm{e}^{v_{k}}\right)\right] .
\end{aligned}
$$

Hence it is easy to see that $J^{k}\left(v_{k}, \phi_{k}\right)$, the Jacobian of the above transformation, satisfies $C_{1} \leq\left|J^{k}\left(v_{k}, \phi_{k}\right)\right| \leq C_{2}$ for all $\left(v_{k}, \phi_{k}\right) \in \hat{S}_{\mu}^{k}$, for all $0<\mu \leq \rho$.

We now need the fundamental regularity result from [2], viz. Theorem 2.1 which we state as follows:

If $f \in H_{\beta}^{m, 0}(\Omega), g^{[j]} \in H_{\beta}^{m+\frac{3}{2}-j, \frac{3}{2}-j}\left(\Gamma^{[j]}\right), j=0,1,0<\beta_{i}<1, \beta_{i}>\beta_{i}^{\star}$ and $m \geq 0$, then the solution of (2.3) exists in $H_{\beta}^{m+2,2}(\Omega)$ and

$$
\|u\|_{H_{\beta}^{m+2,2(\Omega)}} \leq C_{m}\left(\|f\|_{H_{\beta}^{m, 0}(\Omega)}+\sum_{j=0}^{1} \| g_{H_{\beta}^{[j]} \|_{\frac{3}{2}-j, \frac{3}{2}-j} \Gamma_{\left[\Gamma^{[j]}\right)}}\right) .
$$

Let us define $\alpha_{i}=1-\beta_{i}^{\star}$.

We now state the differentiability estimates for the solution $u$ of $(2.3)$ which will be needed in this paper.

\section{PROPOSITION 2.1.}

Let $1-\alpha_{k}>0$. Then for $\lambda_{k}<\alpha_{k}$,

$$
\begin{aligned}
& \int_{\psi_{l}^{k}}^{\psi_{u}^{k}} \int_{-\infty}^{\ln \mu} \sum_{|\varepsilon| \leq m}\left|D_{v_{k}}^{\varepsilon_{1}} D_{\phi_{k}}^{\varepsilon_{2}}\left(u-u\left(A_{k}\right)\right)\right|^{2} \mathrm{e}^{-2 \lambda_{k} v_{k}} \mathrm{~d} v_{k} \mathrm{~d} \phi_{k} \\
& \quad \leq \mu^{2 \gamma_{k}}\left(C d^{m-2}(m-2) !\right)^{2}
\end{aligned}
$$

for $0<\mu \leq \rho$ with $\gamma_{k}<\alpha_{k}-\lambda_{k}$. If $1-\alpha_{k}<0$ then for $\lambda_{k}<1 / 2$, (2.11) remains valid for $0<\mu \leq \rho$ with $\gamma_{k}=1 / 2$.

The proposition can be proved in the same way as Theorem 2.1 of [6].

\section{The stability estimate}

Let

$$
\mathscr{L}(u)=-\sum_{i, j=1}^{2}\left(a_{i, j} u_{x_{j}}\right)_{x_{i}}+\sum_{i=1}^{2} b_{i} u_{x_{i}}+c u
$$

be a strongly elliptic operator. We now consider the following mixed boundary value problem:

$$
\begin{aligned}
& \mathscr{L} u=f \quad \text { in } \Omega, \\
& \bar{\gamma}_{0} u=\left.u\right|_{\Gamma^{[0]}}=g^{[0]} \text { and } \\
& \bar{\gamma}_{1} u=\left.\left(\frac{\partial u}{\partial N}\right)_{A}\right|_{\Gamma^{[1]}}=g^{[1]} .
\end{aligned}
$$


Here the conormal derivative $\bar{\gamma}_{1} u$ is defined as follows. Let $\gamma_{i} \subseteq \Gamma^{[1]}$ and let $N=\left(N_{1}, N_{2}\right)^{T}$ denote the unit outward normal at a point on $\gamma_{i}$. Then

$$
\bar{\gamma}_{1} u=\left(\frac{\partial u}{\partial N}\right)_{A}=\sum_{i, j=1}^{2} N_{i} a_{i, j} u_{x_{j}} .
$$

Moreover, let the bilinear form induced by the operator $\mathscr{L}$ satisfy the inf-sup conditions.

We can now state the regularity result Theorem 2.1 of [2] as follows:

Let $u$ be the solution to (3.2). Then

$$
\|u\|_{H_{\beta}^{k+2,2}(\Omega)} \leq C_{k}\left(\|f\|_{H_{\beta}^{k, 0}(\Omega)}+\sum_{j=0}^{1}\left\|g^{[j]}\right\|_{H_{\beta}^{k+\frac{3}{2}-j, \frac{3}{2}-j}\left(\Gamma^{[j]}\right)}\right) .
$$

The above estimate for $k=0$ is used to prove the stability estimate Theorem 3.1.

We remark that in Theorem 5.2 of [9], Guo and Babuska have extended the above regularity result to elliptic systems. Hence the method applies to elliptic systems too.

Divide the polygonal domain $\Omega$ into $p$ sectors $\Omega^{1}, \Omega^{2}, \ldots, \Omega^{p}$ and a remaining portion $\Omega^{p+1}$. Further divide each of these subdomains into still smaller elements

$$
\left\{\Omega_{i, j}^{k}, 1 \leq i \leq I_{k, j}, 1 \leq j \leq M, 1 \leq k \leq p\right\} .
$$

Let

$$
\Omega^{p+1}=\left\{\Omega_{i, j}^{k}: 1 \leq k \leq p, M<j \leq J_{k}, 1 \leq i \leq I_{k, j}\right\} .
$$

We shall relabel the elements of $\Omega^{p+1}$ and write

$$
\Omega^{p+1}=\left\{\Omega_{l}^{p+1}: 1 \leq l \leq L\right\} .
$$

Now define the space of spectral element functions $\Pi^{M, W}=\left\{\left\{u_{i, j}^{k}\left(v_{k}, \phi_{k}\right)\right\}_{i, j, k}\right.$, $\left.\left\{u_{l}^{p+1}(\xi, \eta)\right\}_{l}\right\}$, where $u_{i, 1}^{k}=h_{k}$ a constant for all $i$ and

$$
u_{i, j}^{k}\left(v_{k}, \phi_{k}\right)=\sum_{r=1}^{W_{j}} \sum_{s=1}^{W_{j}} g_{r, s} v_{k}^{r} \phi_{k}^{s}, \quad 1<j \leq M .
$$

Here $1 \leq W_{j} \leq W$. Moreover there is an analytic mapping $M_{l}^{p+1}$ from the master square $S=(-1,1)^{2}$ to $\Omega_{l}^{p+1}$. We define

$$
u_{l}^{p+1}\left(M_{l}^{p+1}(\xi, \eta)\right)=\sum_{r=1}^{W} \sum_{s=1}^{W} g_{r, s} \xi^{r} \eta^{s} .
$$

Let $w \in H_{\beta}^{2,2}(\Omega)$. Now for $1 \leq j \leq M$,

$$
\int_{\Omega_{i, j}^{k}} r_{k}^{2 \beta_{k}}|\mathscr{L} w|^{2} \mathrm{~d} x=\int_{\tilde{\Omega}_{i, j}^{k}} r_{k}^{2\left(-1+\beta_{k}\right)}\left|\tilde{\mathscr{L}}^{k} w\right|^{2} \mathrm{~d} \tau_{k} \mathrm{~d} \theta_{k}
$$

Here $\tilde{\Omega}_{i, j}^{k}$ is the image of $\Omega_{i, j}^{k}$ in $\left(\tau_{k}, \theta_{k}\right)$ coordinates and $\tilde{\mathscr{L}}^{k} w=r_{k}^{2} \mathscr{L} w$. It has been shown in [7] that if we let $y_{1}=\tau_{k}$ and $y_{2}=\theta_{k}$ then

$$
\tilde{\mathscr{L}}^{k} w=-\sum_{i, j=1}^{2} \frac{\partial}{\partial y_{i}}\left(\tilde{a}_{i, j}^{k} \frac{\partial w}{\partial y_{j}}\right)+\sum_{i=1}^{2} \tilde{b}_{i}^{k} w_{y_{i}}+\tilde{c}^{k} w
$$


Let $O^{k}$ denote the matrix

$$
O^{k}=\left[\begin{array}{cc}
\cos \theta_{k} & -\sin \theta_{k} \\
\sin \theta_{k} & \cos \theta_{k}
\end{array}\right]
$$

and

$$
\tilde{A}^{k}=\left[\begin{array}{ll}
\tilde{a}_{1,1}^{k} & \tilde{a}_{1,2}^{k} \\
\tilde{a}_{2,1}^{k} & \tilde{a}_{2,2}^{k}
\end{array}\right] .
$$

Then $\tilde{A}^{k}=\left(O^{k}\right)^{T} A O^{k}$.

Let $J^{k}\left(v_{k}, \phi_{k}\right)$ denote the Jacobian of the map $M^{k}\left(v_{k}, \phi_{k}\right)$ defined in $\S 2$. Then for $1<$ $j \leq M$,

$$
\int_{\Omega_{i, j}^{k}} r_{k}^{2 \beta_{k}}|\mathscr{L} w|^{2} \mathrm{~d} x=\int_{\hat{\Omega}_{i, j}^{k}} \mathrm{e}^{-2\left(1-\beta_{k}\right) v_{k}}\left|\mathscr{L}_{i, j}^{k} w\left(v_{k}, \phi_{k}\right)\right|^{2} \mathrm{~d} v_{k} \mathrm{~d} \phi_{k}
$$

Here $\hat{\Omega}_{i, j}^{k}$ is the image of $\Omega_{i, j}^{k}$ in $\left(v_{k}, \phi_{k}\right)$ variables and

$$
\mathscr{L}_{i, j}^{k} w=\sqrt{J^{k}} \tilde{\mathscr{L}}^{k} w
$$

Now

$$
\begin{aligned}
\mathscr{L}_{i, j}^{k} w\left(v_{k}, \phi_{k}\right)= & A_{i, j}^{k} w_{v_{k} v_{k}}+2 B_{i, j}^{k} w_{v_{k} \phi_{k}}+C_{i, j}^{k} w_{\phi_{k} \phi_{k}} \\
& +D_{i, j}^{k} w_{v_{k}}+E_{i, j}^{k} w_{\phi_{k}}+F_{i, j}^{k} w .
\end{aligned}
$$

Let $\hat{A}_{i, j}^{k}$ be the polynomial approximation of $A_{i, j}^{k}$, of degree $W_{j}$ in $v_{k}$ and $\phi_{k}$ separately, as defined in Theorem 4.46 of [12]. Now we define a differential operator with polynomial coefficients $\left(\mathscr{L}_{i, j}^{k}\right)^{a}$, which is an approximation to $\mathscr{L}_{i, j}^{k}$ as follows:

$$
\begin{aligned}
\left(\mathscr{L}_{i, j}^{k}\right)^{a} w= & \hat{A}_{i, j}^{k} w_{v_{k} v_{k}}+2 \hat{B}_{i, j}^{k} w_{v_{k} \phi_{k}}+\hat{C}_{i, j}^{k} w_{\phi_{k} \phi_{k}}+\hat{D}_{i, j}^{k} w_{v_{k}} \\
& +\hat{E}_{i, j}^{k} w_{\phi_{k}}+\hat{F}_{i, j}^{k} w .
\end{aligned}
$$

Let $\lambda_{k}=1-\beta_{k}$. Then for $1<j \leq M$,

$$
\begin{aligned}
& \left.\left|\int_{\hat{\Omega}_{i, j}^{k}}\right| \mathscr{L}_{i, j}^{k} w\left(v_{k}, \phi_{k}\right)\right|^{2} \mathrm{e}^{-2 \lambda_{k} v_{k}} \mathrm{~d} v_{k} \mathrm{~d} \phi_{k} \\
& \quad-\int_{\hat{\Omega}_{i, j}^{k}}\left|\left(\mathscr{L}_{i, j}^{k}\right)^{a} w\left(v_{k}, \phi_{k}\right)\right|^{2} \mathrm{e}^{-2 \lambda_{k} v_{k}} \mathrm{~d} v_{k} \mathrm{~d} \phi_{k} \mid \\
& \leq \varepsilon_{W}\left(\left(\rho \mu_{k}^{M+1-j}\right)^{-2 \lambda_{k}}\left\|w\left(v_{k}, \phi_{k}\right)-w\left(A_{k}\right)\right\|_{2, \hat{\Omega}_{i, j}^{k}}^{2}\right. \\
& \left.\quad+\left(\rho \mu_{k}^{M+1-j}\right)^{4-2 \lambda_{k}}\left|w\left(A_{k}\right)\right|^{2}\right) .
\end{aligned}
$$

Here $\varepsilon_{W} \rightarrow 0$ as $W \rightarrow \infty$ and, in fact, $\varepsilon_{W}$ is exponentially small in $W$. 
Moreover, if $w\left(v_{k}, \phi_{k}\right)=w\left(A_{k}\right)$, a constant in $\Omega_{i, 1}^{k}$ for $1 \leq i \leq I_{k}$, then

$$
\sum_{i=1}^{I_{k}} \int_{\hat{\Omega}_{i, 1}^{k}}\left|\mathscr{L}_{i, 1}^{k} w\left(v_{k}, \phi_{k}\right)\right|^{2} \mathrm{e}^{-2 \lambda_{k} v_{k}} \mathrm{~d} v_{k} \mathrm{~d} \phi_{k} \leq \varepsilon_{M}\left|w\left(A_{k}\right)\right|^{2} .
$$

Here $\varepsilon_{M} \rightarrow 0$ as $M \rightarrow \infty$ and $\varepsilon_{M}$ is exponentially small in $M$.

Hence we conclude that if $w\left(v_{k}, \phi_{k}\right)=w\left(A_{k}\right)$, a constant in $\Omega_{i, 1}^{k}$ for $1 \leq i \leq I_{k}$, then

$$
\begin{aligned}
& \sum_{i=1}^{I_{k}} \sum_{j=1}^{M} \int_{\hat{\Omega}_{i, j}^{k}}\left|\mathscr{L}_{i, j}^{k} w_{i, j}^{k}\left(v_{k}, \phi_{k}\right)\right|^{2} \mathrm{e}^{-2 \lambda_{k} v_{k}} \mathrm{~d} v_{k} \mathrm{~d} \phi_{k} \\
& \leq C\left(\sum_{i=1}^{I_{k}} \sum_{j=2}^{M}\left(\rho \mu_{k}^{M+1-j}\right)^{-2 \lambda_{k}}\left(\int_{\hat{\Omega}_{i, j}^{k}}\left|\left(\mathscr{L}_{i, j}^{k}\right)^{a} w_{i, j}^{k}\left(v_{k}, \phi_{k}\right)\right|^{2} \mathrm{~d} v_{k} \mathrm{~d} \phi_{k}\right)\right) \\
& \quad+\varepsilon_{W}\left(\sum_{i=1}^{I_{k}} \sum_{j=2}^{M}\left(\rho \mu_{k}^{M+1-j}\right)^{-2 \lambda_{k}}\left\|w_{i, j}^{k}-w\left(A_{k}\right)\right\|_{2, \hat{\Omega}_{i, j}^{k}}^{2}+\left|w\left(A_{k}\right)\right|^{2}\right) \\
& \quad+\varepsilon_{M}\left|w\left(A_{k}\right)\right|^{2} .
\end{aligned}
$$

Here $C$ is a constant.

Now

$$
\int_{\Omega_{l}^{p+1}}|\mathscr{L} w|^{2} \mathrm{~d} x_{1} \mathrm{~d} x_{2}=\int_{S}\left|\mathscr{L} w_{l}^{p+1}\right|^{2} J_{l}^{p+1} \mathrm{~d} \xi \mathrm{d} \eta
$$

Here $J_{l}^{p+1}(\xi, \eta)$ is the Jacobian of the mapping $M_{l}^{p+1}$ from $S$ to $\Omega_{l}^{p+1}$. Let $\mathscr{L}_{l}^{p+1}(\xi, \eta)=$ $\mathscr{L}(\xi, \eta) \sqrt{J_{l}^{p+1}}$. Once more we can define $\left(\mathscr{L}_{l}^{p+1}\right)^{a}$, a differential operator which is an approximation to $\mathscr{L}_{l}^{p+1}$ in which the coefficients of $\mathscr{L}_{l}^{p+1}$ are replaced by polynomial approximations. It can be shown as before that

$$
\begin{aligned}
& \sum_{l=1}^{L} \int_{\Omega_{l}^{p+1}}|\mathscr{L} w|^{2} \mathrm{~d} x_{1} \mathrm{~d} x_{2} \\
& \quad \leq C \sum_{l=1}^{L} \int_{S}\left|\left(\mathscr{L}_{l}^{p+1}\right)^{a} w_{l}^{p+1}(\xi, \eta)\right|^{2} \mathrm{~d} \xi \mathrm{d} \eta+\varepsilon_{W} \sum_{l=1}^{L}\left\|w_{l}^{p+1}(\xi, \eta)\right\|_{2, S}^{2} .
\end{aligned}
$$

Here $C$ is a constant and $\varepsilon_{W} \rightarrow 0$ as $W \rightarrow \infty$. In fact, $\varepsilon_{W}$ is exponentially small in $W$.

We now prove a result which we shall need in the sequel.

Lemma 3.1. Let $\omega \in H_{\beta}^{2,2}(\Omega)$. Then there exists a constant $C$ such that

$$
\begin{gathered}
\frac{1}{C}\left(\sum _ { k = 1 } ^ { p } \left(\left|\omega\left(A_{k}\right)\right|^{2}+\sum_{|\alpha| \leq 2} \int_{\hat{\Omega}^{k}}\left|D_{v_{k}, \phi_{k}}^{\alpha}\left(\omega\left(v_{k}, \phi_{k}\right)-\omega\left(A_{k}\right)\right)\right|^{2}\right.\right. \\
\left.\left.\quad \times \mathrm{e}^{-2 \lambda_{k} v_{k}} \mathrm{~d} v_{k} \mathrm{~d} \phi_{k}\right)+\left\|\omega\left(x_{1}, x_{2}\right)\right\|_{H^{2}\left(\Omega^{p+1}\right)}^{2}\right)
\end{gathered}
$$




$$
\begin{aligned}
\leq & \|\omega\|_{H_{\beta}^{2,2}(\Omega)}^{2} \\
\leq & C\left(\sum _ { k = 1 } ^ { p } \left(\left|\omega\left(A_{k}\right)\right|^{2}+\sum_{|\alpha| \leq 2} \int_{\hat{\Omega}^{k}}\left|D_{v_{k}, \phi_{k}}^{\alpha}\left(\omega\left(v_{k}, \phi_{k}\right)-\omega\left(A_{k}\right)\right)\right|^{2}\right.\right. \\
& \left.\left.\times \mathrm{e}^{-2 \lambda_{k} v_{k}} \mathrm{~d} v_{k} \mathrm{~d} \phi_{k}\right)+\left\|\omega\left(x_{1}, x_{2}\right)\right\|_{H^{2}\left(\Omega^{p+1}\right)}^{2}\right)
\end{aligned}
$$

Here $\lambda_{k}=1-\beta_{k}$.

Proof. Let $\psi_{k} \in C_{0}^{\infty}(R)$ such that $\psi_{k}\left(r_{k}\right)=1$ for $r_{k} \leq \rho$ and $\psi_{k}\left(r_{k}\right)=0$ for $r_{k} \geq \rho^{1}$ for $k=1,2, \ldots, p$. Here $\rho^{1}>\rho$ is chosen so that $\Omega_{\rho^{1}}^{k}=\left\{\left(x_{1}, x_{2}\right): r_{k} \leq \rho^{1}\right\}$ have the property that $\Omega_{\rho^{1}}^{k} \cap \Omega_{\rho^{1}}^{l}=\emptyset$ if $k \neq l$. We define $\omega_{k}=\omega \psi_{k}$ for $k=1, \ldots, p$ and $\omega_{0}=1-\sum_{k=1}^{p} \omega_{k}$. Then $\omega_{k} \in H_{\beta}^{2,2}(\Omega)$ for $k=1, \ldots, p$.

Now by Lemma 2.1 of $[3], H_{\beta}^{2,2}(\Omega) \subseteq C(\bar{\Omega})$ with continuous injection. Hence we conclude that

$$
\sum_{k=1}^{p}\left|\omega_{k}\left(A_{k}\right)\right|^{2} \leq C \sum_{k=1}^{p}\left\|\omega_{k}\right\|_{H_{\beta}^{2,2}(\Omega)}^{2}
$$

Therefore

$$
\sum_{k=1}^{p}\left|\omega\left(A_{k}\right)\right|^{2} \leq C \sum_{k=1}^{p}\|\omega\|_{H_{\beta}^{2,2}(\Omega)}^{2}
$$

We now cite Lemma 2.2 of [4]. Let $u \in H_{\beta}^{2,2}(\Omega)$. Then

(i)

$$
\sum_{|\alpha|=1}\left\|D^{\alpha} u \Phi_{\beta-1}\right\|_{L^{2}(\Omega)} \leq C\|u\|_{H_{\beta}^{2,2}(\Omega)} .
$$

(ii) Let $u\left(A_{i}\right)=0$, for $i=1, \ldots, p$. Then

$$
\left\|u \Phi_{\beta-2}\right\|_{L^{2}(\Omega)} \leq C\|u\|_{H_{\beta}^{2,2}(\Omega)} .
$$

From (i) we obtain

$$
\sum_{k=1}^{p} \int_{\hat{\Omega}^{k}} \sum_{|\alpha|=1}\left|D_{v_{k}, \phi_{k}}^{\alpha} \omega\left(v_{k}, \phi_{k}\right)\right|^{2} \mathrm{e}^{-2\left(1-\beta_{k}\right) v_{k}} \mathrm{~d} v_{k} \mathrm{~d} \phi_{k} \leq C\left(\|\omega\|_{H_{\beta}^{2,2}(\Omega)}^{2}\right) .
$$

Here $C$ is a generic constant. Now using (ii) we get

$$
\begin{gathered}
\int_{\Omega}\left|\omega_{k}\left(v_{k}, \phi_{k}\right)-\omega\left(A_{k}\right) \psi_{k}\right|^{2} \mathrm{e}^{-2\left(1-\beta_{k}\right) v_{k}} \mathrm{~d} v_{k} \mathrm{~d} \phi_{k} \\
\leq C\left(\left\|\omega_{k}\right\|_{H_{\beta}^{2,2}(\Omega)}^{2}+\left|\omega\left(A_{k}\right)\right|^{2}\right) .
\end{gathered}
$$


Hence

$$
\sum_{k=1}^{p} \int_{\hat{\Omega}^{k}}\left|\omega\left(v_{k}, \phi_{k}\right)-\omega\left(A_{k}\right)\right|^{2} \mathrm{e}^{-2\left(1-\beta_{k}\right) v_{k}} \mathrm{~d} v_{k} \mathrm{~d} \phi_{k} \leq C\|\omega\|_{H_{\beta}^{2,2}(\Omega)}^{2} .
$$

Finally,

$$
\sum_{k=1}^{p} \int_{\hat{\Omega}^{k}} \sum_{|\alpha|=2}\left|D_{v_{k}, \phi_{k}}^{\alpha} \omega\left(v_{k}, \phi_{k}\right)\right|^{2} \mathrm{e}^{-2\left(1-\beta_{k}\right) v_{k}} \mathrm{~d} v_{k} \mathrm{~d} \phi_{k} \leq C\|\omega\|_{H_{\beta}^{2,2}(\Omega)}^{2} .
$$

Combining the estimates (3.10)-(3.13) we get (3.9).

We now introduce some notation which is needed to state the stability estimate Theorem 3.1 which is the main result of this section.

Let $\gamma_{s}$ be a side common to the elements $\Omega_{m}^{p+1}$ and $\Omega_{n}^{p+1}$ and let $\gamma_{s} \subseteq \Omega^{p+1}$. We may assume that $\gamma_{s}$ is the image of $\eta=-1$ under the mapping $M_{m}^{p+1}$ which maps $S$ to $\Omega_{m}^{p+1}$ and also the image of $\eta=1$ under the mapping $M_{n}^{p+1}$ which maps $S$ to $\Omega_{n}^{p+1}$. By the chain rule

$$
\begin{aligned}
& \left(u_{m}^{p+1}\right)_{x_{1}}=\left(u_{m}^{p+1}\right)_{\xi} \xi_{x_{1}}+\left(u_{m}^{p+1}\right)_{\eta} \eta_{x_{1}}, \quad \text { and } \\
& \left(u_{m}^{p+1}\right)_{x_{2}}=\left(u_{m}^{p+1}\right)_{\xi} \xi_{x_{2}}+\left(u_{m}^{p+1}\right)_{\eta} \eta_{x_{2}} .
\end{aligned}
$$

Now let $\hat{\xi}_{x_{1}}$ denote the polynomial approximation of $\xi_{x_{1}}(\xi, \eta)$, of degree $W$ in $\xi$ and $\eta$ separately, as defined in Theorem 4.46 of [12]. In the same way $\hat{\eta}_{x_{1}}, \hat{\xi}_{x_{2}}$ and $\hat{\eta}_{x_{2}}$ can be defined. We now define

$$
\begin{aligned}
& \left(u_{m}^{p+1}\right)_{x_{1}}^{a}=\left(u_{m}^{p+1}\right)_{\xi} \hat{\xi}_{x_{1}}+\left(u_{m}^{p+1}\right)_{\eta} \hat{\eta}_{x_{1}}, \quad \text { and } \\
& \left(u_{m}^{p+1}\right)_{x_{2}}^{a}=\left(u_{m}^{p+1}\right)_{\xi} \hat{\xi}_{x_{2}}+\left(u_{m}^{p+1}\right)_{\eta} \hat{\eta}_{x_{2}} .
\end{aligned}
$$

Let

$$
\begin{aligned}
\left\|\left[u^{p+1}\right]\right\|_{0, \gamma_{s}}^{2} & =\left\|u_{m}^{p+1}(\xi,-1)-u_{n}^{p+1}(\xi, 1)\right\|_{0, I}^{2}, \\
\left\|\left[\left(u_{x_{1}}^{p+1}\right)^{a}\right]\right\|_{1 / 2, \gamma_{s}}^{2} & =\left\|\left(u_{m}^{p+1}\right)_{x_{1}}^{a}(\xi,-1)-\left(u_{n}^{p+1}\right)_{x_{1}}^{a}(\xi, 1)\right\|_{1 / 2, I}^{2}, \quad \text { and } \\
\left\|\left[\left(u_{x_{2}}^{p+1}\right)^{a}\right]\right\|_{1 / 2, \gamma_{s}}^{2} & =\left\|\left(u_{m}^{p+1}\right)_{x_{2}}^{a}(\xi,-1)-\left(u_{n}^{p+1}\right)_{x_{2}}^{a}(\xi, 1)\right\|_{1 / 2, I}^{2} .
\end{aligned}
$$

Here $I=(-1,1)$. Next, let $\gamma_{s} \subseteq \Gamma^{[0]} \cap \partial \Omega^{p+1}$ and let $\gamma_{s}$ be the image of $\eta=-1$ under the mapping $M_{m}^{p+1}$ which maps $S$ to $\Omega_{m}^{p+1}$. We can define $\left(\frac{\partial u_{m}^{p+1}}{\partial T}\right)^{a}$, an approximation to $\frac{\partial u^{p+1}}{\partial T}$ as before. Let

$$
\begin{aligned}
& \left\|u^{p+1}\right\|_{0, \gamma_{s}}^{2}+\left\|\left(\frac{\partial u^{p+1}}{\partial T}\right)^{a}\right\|_{1 / 2, \gamma_{s}}^{2} \\
& =\left\|u_{m}^{p+1}(\xi,-1)\right\|_{0, I}^{2}+\left\|\left(\frac{\partial u_{m}^{p+1}}{\partial T}\right)^{a}(\xi,-1)\right\|_{1 / 2, I}^{2} .
\end{aligned}
$$

In the same way, if $\gamma_{s} \subseteq \Gamma^{[1]} \cap \partial \Omega^{p+1},\left\|\left(\frac{\partial u^{p+1}}{\partial N}\right)_{A}^{a}\right\|_{1 / 2, \gamma_{s}}^{2}$ can be defined. 
Let $\gamma_{s} \subseteq \Gamma^{[1]} \cap \partial \Omega^{k}$ for $1 \leq k \leq p$. Let $\tilde{\gamma}_{s}$ be the image of $\gamma_{s}$ in $\left(\tau_{k}, \theta_{k}\right)$ coordinates and $\hat{\gamma}_{s}$ be the image of $\gamma_{s}$ in $\left(v_{k}, \phi_{k}\right)$ coordinates. Let $\left(n_{1}, n_{2}\right)$ be the normal at a point $\tilde{p}$ on $\tilde{\gamma}_{s}$. Define

$$
\left(\frac{\partial u^{k}}{\partial n}\right)_{\tilde{A}^{k}}=\sum_{i, j=1}^{2} n_{i} \tilde{a}_{i, j}^{k} \frac{\partial u^{k}}{\partial y_{j}} .
$$

Now $\hat{\gamma}_{s}$ is a portion of the straight line $\phi_{k}=\alpha$, where $\alpha$ is a constant. Let $\left(\frac{\partial u^{k}}{\partial n}\right)_{\tilde{A}^{k}}^{a}$, denote an approximation to $\left(\frac{\partial u^{k}}{\partial n}\right)_{\tilde{A}^{k}}$ as before, and using this $\left\|\left(\frac{\partial u^{k}}{\partial n}\right)_{\tilde{A}^{k}}^{a}\right\|_{1 / 2, \gamma_{s}}^{2}$ can be defined. Let $\gamma_{s} \subseteq \bar{\Omega}^{k}$. Define

$$
d\left(A_{k}, \gamma_{s}\right)=\inf _{x \in \gamma_{s}}\left\{\operatorname{distance}\left(A_{k}, x\right)\right\}
$$

Let

$$
\begin{aligned}
\mathscr{V}_{\text {vertices }}^{M, W} & \left(\left\{u_{i, j}^{k}\left(v_{k}, \phi_{k}\right)\right\}_{i, j, k},\left\{u_{l}^{p+1}(\xi, \eta)\right\}_{l}\right) \\
= & \sum_{k=1}^{p} \sum_{j=2}^{M} \sum_{i=1}^{I_{k}}\left(\rho \mu_{k}^{M+1-j}\right)^{-2 \lambda_{k}}\left\|\left(\mathscr{L}_{i, j}^{k}\right)^{a} u_{i, j}^{k}\left(v_{k}, \phi_{k}\right)\right\|_{0, \hat{\Omega}_{i, j}^{k}}^{2} \\
& +\sum_{k=1}^{p} \sum_{\gamma_{s} \subseteq \Omega^{k} \cup B_{\rho}^{k}, \mu\left(\hat{\gamma}_{s}\right)<\infty} d\left(A_{k}, \gamma_{s}\right)^{-2 \lambda_{k}} \\
& \times\left(\left\|\left[u^{k}\right]\right\|_{0, \hat{\gamma}_{s}}^{2}+\left\|\left[\left(u_{v_{k}}^{k}\right)^{a}\right]\right\|_{1 / 2, \hat{\gamma}_{s}}^{2}+\left\|\left[\left(u_{\phi_{k}}^{k}\right)^{a}\right]\right\|_{1 / 2, \hat{\gamma}_{s}}^{2}\right) \\
& +\sum_{l \in \mathscr{D}} \sum_{k=l-1}^{l}\left(\left|h_{k}\right|^{2}+\sum_{\gamma_{s} \subseteq \partial \Omega^{k} \cap \Gamma_{l}, \mu\left(\hat{\gamma}_{s}\right)<\infty} d\left(A_{k}, \gamma_{s}\right)^{-2 \lambda_{k}}\right. \\
& \left.\times\left(\left\|u^{k}-h_{k}\right\|_{0, \hat{\gamma}_{s}}^{2}+\left\|u_{v_{k}}^{k}\right\|_{1 / 2, \gamma_{s}}^{2}\right)\right) \\
& +\sum_{l \in \mathscr{N}} \sum_{k=l-1}^{l} \sum_{\gamma_{s} \subseteq \partial \Omega^{k} \cap \Gamma_{l}, \mu\left(\hat{\gamma}_{s}\right)<\infty} d\left(A_{k}, \gamma_{s}\right)^{-2 \lambda_{k}}\left\|\left(\frac{\partial u^{k}}{\partial n}\right)_{\tilde{A}^{k}}^{a}\right\|_{1 / 2, \hat{\gamma}_{s}}^{2} .
\end{aligned}
$$

Here $\left\{\left\{u_{i, j}^{k}\left(v_{k}, \phi_{k}\right)\right\}_{i, j, k},\left\{u_{l}^{p+1}(\xi, \eta)\right\}_{l}\right\} \in \Pi^{M, W}$ and $u_{i, 1}^{k}=h_{k}$ for $1 \leq i \leq I_{k}$. Moreover $\mu\left(\hat{\gamma}_{s}\right)$ denotes the measure of $\hat{\gamma}_{s}$. Next, we define

$$
\begin{aligned}
\mathscr{V}_{\text {interior }}^{M, W} & \left(\left\{u_{i, j}^{k}\left(v_{k}, \phi_{k}\right)\right\}_{i, j, k},\left\{u_{l}^{p+1}(\xi, \eta)\right\}_{l}\right) \\
= & \sum_{l=1}^{L}\left\|\left(\mathscr{L}_{l}^{p+1}\right)^{a} u_{l}^{p+1}(\xi, \eta)\right\|_{0, S}^{2} \\
& +\sum_{\gamma_{s} \subseteq \Omega^{p+1}}\left(\left\|\left[u^{p+1}\right]\right\|_{0, \gamma_{s}}^{2}+\left\|\left[\left(u_{x_{1}}^{p+1}\right)^{a}\right]\right\|_{1 / 2, \gamma_{s}}^{2}+\left\|\left[\left(u_{x_{2}}^{p+1}\right)^{a}\right]\right\|_{1 / 2, \gamma_{s}}^{2}\right)
\end{aligned}
$$




$$
\begin{aligned}
& +\sum_{l \in \mathscr{D}} \sum_{\gamma_{s} \subseteq \partial \Omega^{p+1} \cap \Gamma_{l}}\left(\left\|u^{p+1}\right\|_{0, \gamma_{s}}^{2}+\left\|\left(\frac{\partial u^{p+1}}{\partial T}\right)^{a}\right\|_{1 / 2, \gamma_{s}}^{2}\right) \\
& +\sum_{l \in \mathscr{N}} \sum_{\gamma_{s} \subseteq \partial \Omega^{p+1} \cap \Gamma_{l}}\left\|\left(\frac{\partial u^{p+1}}{\partial N}\right)_{A}^{a}\right\|_{1 / 2, \gamma_{s}}^{2} \cdot
\end{aligned}
$$

Let

$$
\begin{aligned}
\mathscr{V}^{M, W} & \left(\left\{u_{i, j}^{k}\left(v_{k}, \phi_{k}\right)\right\}_{i, j, k},\left\{u_{l}^{p+1}(\xi, \eta)\right\}_{l}\right) \\
= & \mathscr{V}_{\text {vertices }}^{M, W}\left(\left\{u_{i, j}^{k}\left(v_{k}, \phi_{k}\right)\right\}_{i, j, k},\left\{u_{l}^{p+1}(\xi, \eta)\right\}_{l}\right) \\
& +\mathscr{V}_{\text {interior }}^{M, W}\left(\left\{u_{i, j}^{k}\left(v_{k}, \phi_{k}\right)\right\}_{i, j, k},\left\{u_{l}^{p+1}(\xi, \eta)\right\}_{l}\right) .
\end{aligned}
$$

We can now state the main result of this section.

Theorem 3.2. For $M$ and $W$ large enough the estimate

$$
\begin{aligned}
& \sum_{k=1}^{p}\left(\left|h_{k}\right|^{2}+\sum_{i=1}^{I_{k}} \sum_{j=2}^{M}\left(\rho \mu_{k}^{M+1-j}\right)^{-2 \lambda_{k}}\left\|u_{i, j}^{k}\left(v_{k}, \phi_{k}\right)-h_{k}\right\|_{2, \hat{\Omega}_{i, j}^{k}}^{2}\right) \\
& \quad+\sum_{l=1}^{L}\left\|u_{l}^{p+1}(\xi, \eta)\right\|_{2, S}^{2} \\
& \leq C(\ln W)^{2} \mathscr{V}^{M, W}\left(\left\{u_{i, j}^{k}\left(v_{k}, \phi_{k}\right)\right\}_{i, j, k},\left\{u_{l}^{p+1}(\xi, \eta)\right\}_{l}\right)
\end{aligned}
$$

holds. Here C is a constant.

Proof. By Lemma 7.1 there exist $\left\{\left\{v_{i, j}^{k}\left(v_{k}, \phi_{k}\right)\right\}_{i, j, k},\left\{v_{l}^{p+1}(\xi, \eta)\right\}_{l}\right\}$ such that $w$ defined as $w=u+v \in H_{\beta}^{2,2}(\Omega)$. Moreover $v_{i, 1}^{k}=0$ for all $i$ and $k$. Hence by Theorem 2.1 of [2],

$$
\|w\|_{H_{\beta}^{2,2}(\Omega)}^{2} \leq C\left(\|\mathscr{L} w\|_{L_{\beta}(\Omega)}^{2}+\|w\|_{H_{\beta}^{\frac{3}{2}, \frac{3}{2}\left(\Gamma^{[0]}\right)}}^{2}+\left\|\left(\frac{\partial w}{\partial N}\right)_{A}\right\|_{H_{\beta}^{\frac{1}{2}, \frac{1}{2}\left(\Gamma^{[1]}\right)}}^{2}\right)
$$

Now $v_{i, 1}^{k}\left(v_{k}, \phi_{k}\right)=0$ for $1 \leq i \leq I_{k}$. Hence by (3.8),

$$
\begin{aligned}
& \|\mathscr{L} w\|_{L_{\beta}(\Omega)}^{2} \\
& \quad \leq 2\left(\sum_{k=1}^{p} \sum_{j=2}^{M} \sum_{i=1}^{I_{k}}\left\|\left(\mathscr{L}_{i, j}^{k}\right)^{a} u_{i, j}^{k}\left(v_{k}, \phi_{k}\right)\right\|_{0, \hat{\Omega}_{i, j}^{k}}^{2}+\sum_{l=1}^{L}\left\|\left(\mathscr{L}_{l}^{p+1}\right)^{a} u_{l}^{p+1}(\xi, \eta)\right\|_{0, S}^{2}\right)
\end{aligned}
$$




$$
\begin{aligned}
& +C\left(\sum_{k=1}^{p} \sum_{i=1}^{I_{k}} \sum_{j=2}^{M}\left(\rho \mu_{k}^{M+1-j}\right)^{-2 \lambda_{k}}\left\|v_{i, j}^{k}\left(v_{k}, \phi_{k}\right)\right\|_{2, \hat{\Omega}_{i, j}^{k}}^{2}+\sum_{l=1}^{L}\left\|v_{l}^{p+1}(\xi, \eta)\right\|_{2, S}^{2}\right) \\
& +\varepsilon_{W}\left(\sum_{k=1}^{p} \sum_{j=2}^{M} \sum_{i=1}^{I_{k}}\left(\rho \mu_{k}^{M+1-j}\right)^{-2 \lambda_{k}}\left\|u_{i, j}^{k}\left(v_{k}, \phi_{k}\right)-h_{k}\right\|_{2, \hat{\Omega}_{i, j}^{k}}^{2}+\sum_{k=1}^{p}\left|h_{k}\right|^{2}\right) \\
& +\varepsilon_{W}\left(\sum_{l=1}^{L}\left\|u_{l}^{p+1}(\xi, \eta)\right\|_{2, S}^{2}\right)+\varepsilon_{M}\left(\sum_{k=1}^{p}\left|h_{k}\right|^{2}\right) .
\end{aligned}
$$

Now using Lemma 7.2,

$$
\begin{aligned}
& \|w\|_{H_{\beta}^{\frac{3}{2}, \frac{3}{2}}\left(\Gamma^{[0]}\right)}^{2}+\left\|\left(\frac{\partial w}{\partial N}\right)_{A}\right\|_{H_{\beta}^{\frac{1}{2}, \frac{1}{2}}\left(\Gamma^{[1]}\right)}^{2} \\
& \leq C(\ln W)^{2}\left(\sum_{k: \partial \Omega^{k} \cap[0] \neq \emptyset}^{p}\left|h_{k}\right|^{2}+\sum_{l \in \mathscr{D}} \sum_{k=l-1}^{l}\right. \\
& \times \sum_{\gamma_{s} \subseteq \partial \Omega^{k} \cap \Gamma_{l}, \mu\left(\hat{\gamma}_{s}\right)<\infty} d\left(A_{k}, \gamma_{s}\right)^{-2 \lambda_{k}}\left(\left\|u^{k}-h_{k}\right\|_{0, \hat{\gamma}_{s}}^{2}+\left\|u_{v_{k}}^{k}\right\|_{1 / 2, \hat{\gamma}_{s}}^{2}\right) \\
& +\sum_{l \in \mathscr{N}} \sum_{k=l-1}^{l} \sum_{\gamma_{s} \subseteq \partial \Omega^{k} \cap \Gamma_{l}, \mu\left(\hat{\gamma}_{s}\right)<\infty} d\left(A_{k}, \gamma_{s}\right)^{-2 \lambda_{k}}\left\|\left(\frac{\partial u^{k}}{\partial n}\right)_{\tilde{A}^{k}}^{a}\right\|_{1 / 2, \hat{\gamma}_{s}}^{2} \\
& +\sum_{l \in \mathscr{D}} \sum_{\gamma_{s} \subseteq \partial \Omega^{p+1} \cap \Gamma_{l}}\left(\left\|u^{p+1}\right\|_{0, \gamma_{s}}^{2}+\left\|\left(\frac{\partial u^{p+1}}{\partial T}\right)^{a}\right\|_{1 / 2, \gamma_{s}}^{2}\right) \\
& +\sum_{l \in \mathscr{N}} \sum_{\gamma_{s} \subseteq \partial \Omega^{p+1} \cap \Gamma_{l}}\left\|\left(\frac{\partial u^{p+1}}{\partial N}\right)_{A}^{a}\right\|_{1 / 2, \gamma_{s}}^{2} \\
& +\sum_{k=1}^{p} \sum_{\gamma_{s} \subseteq \Omega^{k} \cup B_{\rho}^{k}, \mu\left(\hat{\gamma}_{s}\right)<\infty} d\left(A_{k}, \gamma_{s}\right)^{-2 \lambda_{k}} \\
& \times\left(\left\|\left[u^{k}\right]\right\|_{0, \gamma_{s}}^{2}+\left\|\left[\left(u_{v_{k}}^{k}\right)^{a}\right]\right\|_{1 / 2, \hat{\gamma}_{s}}^{2}+\left\|\left[\left(u_{\phi_{k}}^{k}\right)^{a}\right]\right\|_{1 / 2, \hat{\gamma}_{s}}^{2}\right) \\
& \left.+\sum_{\gamma_{s} \subseteq \Omega^{p+1}}\left(\left\|\left[u^{p+1}\right]\right\|_{0, \gamma_{s}}^{2}+\left\|\left[\left(u_{x_{1}}^{p+1}\right)^{a}\right]\right\|_{1 / 2, \gamma_{s}}^{2}+\left\|\left[\left(u_{x_{2}}^{p+1}\right)^{a}\right]\right\|_{1 / 2, \gamma_{s}}^{2}\right)\right) \\
& +\varepsilon_{W}\left(\sum_{k=1}^{p}\left|h_{k}\right|^{2}+\sum_{k=1}^{p} \sum_{i=1}^{I_{k}} \sum_{j=2}^{M}\left(\rho \mu_{k}^{M+1-j}\right)^{-2 \lambda_{k}} \| u_{i, j}^{k}\left(v_{k}, \phi_{k}\right)\right. \\
& \left.-h_{k}\left\|_{2, \hat{\Omega}_{i, j}^{k}}^{2}+\sum_{l=1}^{L}\right\| u_{l}^{p+1}(\xi, \eta) \|_{2, S}^{2}\right) \text {. }
\end{aligned}
$$


Combining (3.17)-(3.19) we obtain

$$
\begin{aligned}
\|w\|_{H_{\beta}^{2,2}(\Omega)}^{2} \leq & C(\ln W)^{2} \mathscr{V}^{M, W}\left(\left\{u_{i, j}^{k}\left(v_{k}, \phi_{k}\right)\right\}_{i, j, k},\left\{u_{l}^{p+1}(\xi, \eta)\right\}_{l}\right) \\
& +C\left(\sum_{k=1}^{p} \sum_{i=1}^{I_{k}} \sum_{j=2}^{M}\left(\rho \mu_{k}^{M+1-j}\right)^{-2 \lambda_{k}}\left\|v_{i, j}^{k}\left(v_{k}, \phi_{k}\right)\right\|_{2, \hat{\Omega}_{i, j}^{k}}^{2}+\sum_{l=1}^{L}\left\|v_{l}^{p+1}(\xi, \eta)\right\|_{2, S}^{2}\right) \\
& +\varepsilon_{W}\left(\sum_{k=1}^{p} \sum_{j=2}^{M} \sum_{i=1}^{I_{k}}\left(\rho \mu_{k}^{M+1-j}\right)^{-2 \lambda_{k}}\left\|u_{i, j}^{k}\left(v_{k}, \phi_{k}\right)-h_{k}\right\|_{2, \hat{\Omega}_{i, j}^{k}}^{2}+\sum_{k=1}^{p}\left|h_{k}\right|^{2}\right) \\
& +\varepsilon_{W}\left(\sum_{l=1}^{L}\left\|u_{l}^{p+1}(\xi, \eta)\right\|_{2, S}^{2}\right)+\varepsilon_{M}\left(\sum_{k=1}^{p}\left|h_{k}\right|^{2}\right)
\end{aligned}
$$

Now using (3.9),

$$
\begin{aligned}
& \sum_{k=1}^{p}\left(\left|h_{k}\right|^{2}+\sum_{i=1}^{I_{k}} \sum_{j=2}^{M}\left(\rho \mu_{k}^{M+1-j}\right)^{-2 \lambda_{k}}\left\|u_{i, j}^{k}\left(v_{k}, \phi_{k}\right)-h_{k}\right\|_{2, \hat{\Omega}_{i, j}^{k}}^{2}\right) \\
& \quad+\sum_{l=1}^{L}\left\|u_{l}^{p+1}(\xi, \eta)\right\|_{2, S}^{2} \\
& \leq K\left(\|w\|_{H_{\beta}^{2,2}(\Omega)}^{2}+\sum_{k=1}^{p} \sum_{i=1}^{I_{k}} \sum_{j=2}^{M}\left(\rho \mu_{k}^{M+1-j}\right)^{-2 \lambda_{k}}\left\|v_{i, j}^{k}\left(v_{k}, \phi_{k}\right)\right\|_{2, \hat{\Omega} \hat{\Omega}_{i, j}^{k}}^{2}\right. \\
& \left.\quad+\sum_{l=1}^{L}\left\|v_{l}^{p+1}(\xi, \eta)\right\|_{2, S}^{2}\right) .
\end{aligned}
$$

Combining (3.20) and (3.21) gives

$$
\begin{aligned}
& \sum_{k=1}^{p}\left(\left|h_{k}\right|^{2}+\sum_{i=1}^{I_{k}} \sum_{j=2}^{M}\left(\rho \mu_{k}^{M+1-j}\right)^{-2 \lambda_{k}}\left\|u_{i, j}^{k}\left(v_{k}, \phi_{k}\right)-h_{k}\right\|_{2, \hat{\Omega}_{i, j}^{k}}^{2}\right)+\sum_{l=1}^{L}\left\|u_{l}^{p+1}(\xi, \eta)\right\|_{2, S}^{2} \\
& \leq C(\ln W)^{2} \mathscr{V}^{M, W}\left(\left\{u_{i, j}^{k}\left(v_{k}, \phi_{k}\right)\right\}_{i, j, k},\left\{u_{l}^{p+1}(\xi, \eta)\right\}_{l}\right) \\
& \quad+C\left(\sum_{k=1}^{p} \sum_{i=1}^{I_{k}} \sum_{j=2}^{M}\left(\rho \mu_{k}^{M+1-j}\right)^{-2 \lambda_{k}}\left\|v_{i, j}^{k}\left(v_{k}, \phi_{k}\right)\right\|_{2, \hat{\Omega}_{i, j}^{k}}^{2}+\sum_{l=1}^{L}\left\|v_{l}^{p+1}(\xi, \eta)\right\|_{2, S}^{2}\right) \\
& \quad+\varepsilon_{W}\left(\sum_{k=1}^{p} \sum_{j=2}^{M} \sum_{i=1}^{I_{k}}\left(\rho \mu_{k}^{M+1-j}\right)^{-2 \lambda_{k}}\left\|u_{i, j}^{k}\left(v_{k}, \phi_{k}\right)-h_{k}\right\|_{2, \hat{\Omega}_{i, j}^{k}}^{2}+\sum_{k=1}^{p}\left|h_{k}\right|^{2}\right) \\
& \quad+\varepsilon_{W}\left(\sum_{l=1}^{L}\left\|u_{l}^{p+1}(\xi, \eta)\right\|_{2, S}^{2}\right)+\varepsilon_{M}\left(\sum_{k=1}^{p}\left|h_{k}\right|^{2}\right)
\end{aligned}
$$


Now by Lemma 7.1,

$$
\begin{aligned}
& \sum_{k=1}^{p} \sum_{i=1}^{I_{k}} \sum_{j=2}^{M}\left(\rho \mu_{k}^{M+1-j}\right)^{-2 \lambda_{k}}\left\|v_{i, j}^{k}\left(v_{k}, \phi_{k}\right)\right\|_{2, \hat{\Omega}_{i, j}^{k}}^{2}+\sum_{l=1}^{L}\left\|v_{l}^{p+1}(\xi, \eta)\right\|_{2, S}^{2} \\
& \leq C(\ln W)^{2}\left(\sum_{k=1}^{p} \sum_{\gamma_{s} \subseteq \Omega^{k} \cup B_{\rho}^{k}, \mu\left(\hat{\gamma}_{s}\right)<\infty} d\left(A_{k}, \gamma_{s}\right)^{-2 \lambda_{k}}\right. \\
& \times\left(\left\|\left[u^{k}\right]\right\|_{0, \gamma_{s}}^{2}+\left\|\left[\left(u_{v_{k}}^{k}\right)^{a}\right]\right\|_{1 / 2, \gamma_{s}}^{2}+\left\|\left[\left(u_{\phi_{k}}^{k}\right)^{a}\right]\right\|_{1 / 2, \hat{\gamma}_{s}}^{2}\right) \\
&\left.+\sum_{\gamma_{s} \subseteq \Omega^{p+1}}\left(\left\|\left[u^{p+1}\right]\right\|_{0, \gamma_{s}}^{2}+\left\|\left[\left(u_{x_{1}}^{p+1}\right)^{a}\right]\right\|_{1 / 2, \gamma_{s}}^{2}+\left\|\left[\left(u_{x_{2}}^{p+1}\right)^{a}\right]\right\|_{1 / 2, \gamma_{s}}^{2}\right)\right) \\
&+\varepsilon_{W}\left(\sum_{k=1}^{p} \sum_{i=1}^{I_{k}} \sum_{j=2}^{M}\left(\rho \mu_{k}^{M+1-j}\right)^{-2 \lambda_{k}}\left\|u_{i, j}^{k}\left(v_{k}, \phi_{k}\right)-h_{k}\right\|_{2, \hat{\Omega}_{i, j}^{k}}^{2}\right. \\
&\left.+\sum_{l=1}^{L}\left\|u_{l}^{p+1}(\xi, \eta)\right\|_{2, S}^{2}\right) .
\end{aligned}
$$

Combining (3.22) and (3.23) we get the result.

\section{The numerical scheme}

As in $\S 3$,

$$
\hat{\Omega}_{i, j}^{k}=\left\{\left(v_{k}, \phi_{k}\right): v_{j}^{k}<v_{k}<v_{j+1}^{k}, \psi_{i}^{k}<\phi_{k}<\psi_{i+1}^{k}\right\}
$$

for $1 \leq j \leq M, 1 \leq i \leq I_{k, j}, 1 \leq k \leq p$ in $v_{k}$ and $\phi_{k}$ variables.

We now define a nonconforming spectral element representation on each of these subdomains as follows:

$$
u_{i, j}^{k}\left(v_{k}, \phi_{k}\right)=h_{k}, \quad \text { if } j=1,1 \leq i \leq I_{k}, 1 \leq k \leq p
$$

and

$$
u_{i, j}^{k}\left(v_{k}, \phi_{k}\right)=\sum_{m=1}^{W_{j}} \sum_{n=1}^{W_{j}} a_{m, n} v_{k}^{m} \phi_{k}^{n}
$$

for $1<j \leq M, 1 \leq i \leq I_{k, j}, 1 \leq k \leq p$. Here $1 \leq W_{j} \leq W$. Let

$$
\Omega^{p+1}=\left\{\Omega_{l}^{p+1}, 1 \leq l \leq L\right\} .
$$

We define the analytic map $M_{l}^{p+1}$ from the master square $S=(-1,1)^{2}$ to $\Omega_{l}^{p+1}$ and let

$$
u_{l}^{p+1}\left(M_{l}^{p+1}(\xi, \eta)\right)=\sum_{m=1}^{W} \sum_{n=1}^{W} a_{m, n} \xi^{m} \eta^{n}
$$


Let $f_{l}^{p+1}(\xi, \eta)=f\left(X_{l}^{p+1}(\xi, \eta), Y_{l}^{p+1}(\xi, \eta)\right)$ for $1 \leq l \leq L$ and $J_{l}^{p+1}(\xi, \eta)$ denote the Jacobian of the mapping $M_{l}^{p+1}$. Define $F_{l}^{p+1}(\xi, \eta)=f_{l}^{p+1}(\xi, \eta) \sqrt{J_{l}^{p+1}(\xi, \eta)}$ and let $\hat{F}_{l}^{p+1}(\xi, \eta)$ denote the unique polynomial which is the orthogonal projection of $F_{l}^{p+1}(\xi, \eta)$ into the space of polynomials of degree $2 W$ in $\xi$ and $\eta$ with respect to the usual inner product in $H^{2}(S)$.

Next, let the vertex $A_{k}=\left(x_{k}, y_{k}\right)$. As defined in $\S 2$ we have the following relationship between $\left(\tau_{k}, \theta_{k}\right)$ : and $\left(v_{k}, \phi_{k}\right)$ coordinates:

$$
\begin{aligned}
& v_{k}=\tau_{k}, \\
& \theta_{k}=\frac{1}{\left(\psi_{u}^{k}-\psi_{l}^{k}\right)}\left[\left(\phi_{k}-\psi_{l}^{k}\right) f_{1}^{k}\left(\mathrm{e}^{v_{k}}\right)-\left(\phi_{k}-\psi_{u}^{k}\right) f_{0}^{k}\left(\mathrm{e}^{v_{k}}\right)\right] .
\end{aligned}
$$

Define $f^{k}\left(\tau_{k}, \theta_{k}\right)=\mathrm{e}^{2 \tau_{k}} f\left(x_{k}+\mathrm{e}^{\tau_{k}} \cos \theta_{k}, y_{k}+\mathrm{e}^{\tau_{k}} \sin \theta_{k}\right)$ for $1 \leq k \leq p$, and $F_{i, j}^{k}\left(v_{k}, \phi_{k}\right)=$ $f^{k}\left(\tau_{k}, \theta_{k}\right)$ for $\left(v_{k}, \phi_{k}\right) \in \hat{\Omega}_{i, j}^{k}$. Let $\hat{F}_{i, j}^{k}\left(v_{k}, \phi_{k}\right)$ denote the polynomial of degree $2 W_{j}$ in $v_{k}$ and $\phi_{k}$ variables which is the orthogonal projection of $F_{i, j}^{k}\left(v_{k}, \phi_{k}\right)$ into the space of polynomials of degree $2 W_{j}$ in $v_{k}$ and $\phi_{k}$ variables with respect to the usual inner product in $H^{2}\left(\hat{\Omega}_{i, j}^{k}\right)$. Here $2 \leq j \leq M$.

We now consider the boundary condition $u=g_{k}$ on $\Gamma_{k}$ for $k \in \mathscr{D}$ and let $\left(\frac{\partial u}{\partial N}\right)_{A}=g_{k}$ on $\Gamma_{k}$ for $k \in \mathscr{N}$. Define

$l_{1}^{k}\left(v_{k}\right)=\left\{\begin{array}{l}u=g_{k}\left(x_{k}+\mathrm{e}^{v_{k}} \cos \left(f_{0}^{k}\left(\mathrm{e}^{v_{k}}\right)\right), y_{k}+\mathrm{e}^{v_{k}} \sin \left(f_{0}^{k}\left(\mathrm{e}^{v_{k}}\right)\right)\right), \text { for } k \in \mathscr{D}, \\ \left(\frac{\partial u}{\partial n}\right)_{\tilde{A}^{k}}=\mathrm{e}^{v_{k}} g_{k}\left(x_{k}+\mathrm{e}^{v_{k}} \cos \left(f_{0}^{k}\left(\mathrm{e}^{v_{k}}\right)\right), y_{k}+e^{v_{k}} \sin \left(f_{0}^{k}\left(\mathrm{e}^{v_{k}}\right)\right)\right), \\ \text { for } k \in \mathscr{N} .\end{array}\right.$

Let $\hat{l}_{1, j}^{k}\left(v_{k}\right)$ be the orthogonal projection of $l_{1}^{k}\left(v_{k}\right)$ into the space of polynomials of degree $2 W_{j}$ with respect to the usual inner product on $H^{2}\left(v_{j}^{k}, v_{j+1}^{k}\right)$ for $2 \leq j \leq M$.

Consider the boundary condition $u=g_{k}$ on $\Gamma_{k} \cap \partial \Omega^{k-1}$. Define

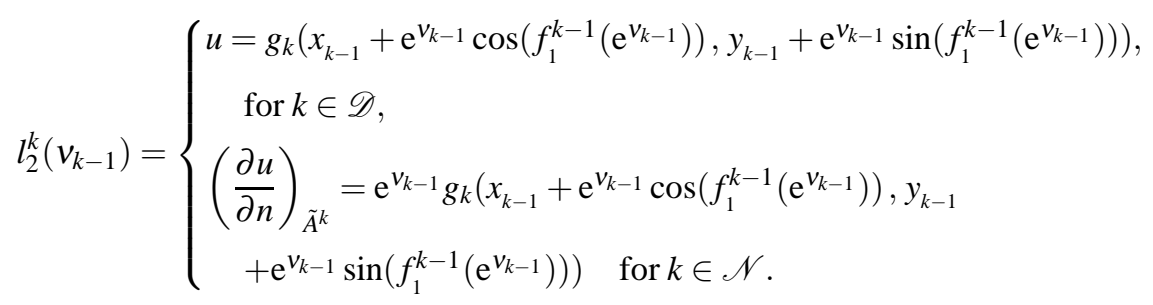

Let $a_{k}=u\left(A_{k}\right)$ if $\gamma_{k}$ or $\gamma_{k+1} \in \mathscr{D}$. We define $\hat{l}_{2, j}^{k}\left(v_{k-1}\right)$ to be the orthogonal projection of $l_{2}^{k}\left(v_{k-1}\right)$ into the space of polynomials of degree $2 W_{j}$ with respect to the usual inner product on $H^{2}\left(v_{j}^{k-1}, v_{j+1}^{k-1}\right)$ for $2 \leq j \leq M$.

Finally, let $\Gamma_{k} \bigcap \partial \Omega_{t}^{p+1}=C_{t}^{k}$ be the image of the mapping $M_{t}^{p+1}$ of $\bar{S}$ onto $\bar{\Omega}_{t}^{p+1}$ corresponding to the side $\xi=-1$. Let $o_{t}^{k}(\eta)=g_{k}\left(X_{t}^{p+1}(-1, \eta), Y_{t}^{p+1}(-1, \eta)\right)$, where $-1 \leq \eta \leq 1$. Define $\hat{o}_{t}^{k}(\eta)$ to be the polynomial of degree $2 W$ which is the orthogonal projection of $o_{t}^{k}(\eta)$ with respect to the usual inner product in $H^{2}(-1,1)$. 
Now we formulate the numerical scheme for problems with mixed boundary conditions.

Let $\left\{\left\{v_{i, j}^{k}\left(v_{k}, \phi_{k}\right)\right\}_{i, j, k},\left\{v_{l}^{p+1}(\xi, \eta)\right\}_{l}\right\} \in \Pi^{M, W}$, the space of spectral element functions. Define the functional

$$
\begin{aligned}
\mathfrak{r}_{\text {vertices }}^{M, W} & \left(\left\{v_{i, j}^{k}\left(v_{k}, \phi_{k}\right)\right\}_{i, j, k},\left\{v_{l}^{p+1}(\xi, \eta)\right\}_{l}\right) \\
= & \sum_{k=1}^{p} \sum_{j=2}^{M} \sum_{i=1}^{I_{k}}\left(\rho \mu_{k}^{M+1-j}\right)^{-2 \lambda_{k}}\left\|\left(\mathscr{L}_{i, j}^{k}\right)^{a} v_{i, j}^{k}\left(v_{k}, \phi_{k}\right)-\hat{F}_{i, j}^{k}\left(v_{k}, \phi_{k}\right)\right\|_{0, \hat{\Omega}_{i, j}^{k}}^{2} \\
& +\sum_{k=1}^{p} \sum_{\gamma_{s} \subseteq \Omega^{k} \cup B_{\rho}^{k}, \mu\left(\hat{\gamma}_{s}\right)<\infty} d\left(A_{k}, \gamma_{s}\right)^{-2 \lambda_{k}} \\
& \times\left(\left\|\left[v^{k}\right]\right\|_{0, \hat{\gamma}_{s}}^{2}+\left\|\left[\left(v_{v_{k}}^{k}\right)^{a}\right]\right\|_{1 / 2, \hat{\gamma}_{s}}^{2}+\left\|\left[\left(v_{\phi_{k}}^{k}\right)^{a}\right]\right\|_{1 / 2, \hat{\gamma}_{s}}^{2}\right) \\
& +\sum_{m \in \mathscr{D}} \sum_{k=m-1}^{m} \sum_{\gamma_{s} \subseteq \partial \Omega^{k} \cap \Gamma_{m}, \mu\left(\hat{\gamma}_{s}\right)<\infty} d\left(A_{k}, \gamma_{s}\right)^{-2 \lambda_{k}}\left(\|\left(v^{k}-h_{k}\right)\right. \\
& \left.-\left(\hat{l}_{m-k+1}^{m}-a_{k}\right)\left\|_{0, \hat{\gamma}_{s}}^{2}+\right\| v_{v_{k}}^{k}-\left(\hat{l}_{m-k+1}^{m}\right)_{v_{k}} \|_{1 / 2, \hat{\gamma}_{s}}^{2}\right) \\
& +\sum_{m \in \mathscr{D}} \sum_{k=m-1}^{m}\left(h_{k}-a_{k}\right)^{2}+\sum_{m \in \mathscr{N}} \sum_{k=m-1}^{m} \\
& \times \sum_{\gamma_{s} \subseteq \partial \Omega^{k} \cap \Gamma_{m}, \mu\left(\hat{\gamma}_{s}\right)<\infty} d\left(A_{k}, \gamma_{s}\right)^{-2 \lambda_{k}}\left\|\left(\frac{\partial v^{k}}{\partial n}\right)_{\tilde{A}^{k}}^{a}-\hat{l}_{m-k+1}^{m}\right\|_{1 / 2, \hat{\gamma}_{s}}^{2}
\end{aligned}
$$

In the above $\mu\left(\hat{\gamma}_{s}\right)$ denotes the measure of $\hat{\gamma}_{s}$.

Next, define

$$
\begin{aligned}
\mathfrak{r}_{\text {interior }}^{M, W} & \left(\left\{v_{i, j}^{k}\left(v_{k}, \phi_{k}\right)\right\}_{i, j, k},\left\{v_{l}^{p+1}(\xi, \eta)\right\}_{l}\right) \\
= & \sum_{l=1}^{L}\left\|\left(\mathscr{L}_{l}^{p+1}\right)^{a} v_{l}^{p+1}(\xi, \eta)-\hat{F}_{l}^{p+1}(\xi, \eta)\right\|_{0, S}^{2} \\
& +\sum_{\gamma_{s} \subseteq \Omega^{p+1}}\left(\left\|\left[v^{p+1}\right]\right\|_{0, \gamma_{s}}^{2}+\left\|\left[\left(v_{x_{1}}^{p+1}\right)^{a}\right]\right\|_{1 / 2, \gamma_{s}}^{2}+\left\|\left[\left(v_{x_{2}}^{p+1}\right)^{a}\right]\right\|_{1 / 2, \gamma_{s}}^{2}\right) \\
& +\sum_{l \in \mathscr{D}} \sum_{\gamma_{s} \subseteq \partial \Omega^{p+1} \cap \Gamma_{l}}\left(\left\|v^{p+1}-\hat{o}^{l}\right\|_{0, \gamma_{s}}^{2}+\left\|\left(\frac{\partial v^{p+1}}{\partial T}\right)^{a}-\left(\frac{\partial \hat{o}^{l}}{\partial T}\right)^{a}\right\|_{1 / 2, \gamma_{s}}^{2}\right) \\
& +\sum_{l \in \mathscr{N}} \sum_{\gamma_{s} \subseteq \partial \Omega^{p+1} \cap \Gamma_{l}}\left\|\left(\frac{\partial v^{p+1}}{\partial N}\right)_{A}^{a}-\hat{o}^{l}\right\|_{1 / 2, \gamma_{s}}^{2} .
\end{aligned}
$$


Let

$$
\begin{aligned}
\mathfrak{r}^{M, W} & \left(\left\{v_{i, j}^{k}\left(v_{k}, \phi_{k}\right)\right\}_{i, j, k},\left\{v_{l}^{p+1}(\xi, \eta)\right\}_{l}\right) \\
= & \mathfrak{r}_{\text {vertices }}^{M, W}\left(\left\{v_{i, j}^{k}\left(v_{k}, \phi_{k}\right)\right\}_{i, j, k},\left\{v_{l}^{p+1}(\xi, \eta)\right\}_{l}\right) \\
& +\mathfrak{r}_{\text {interior }}^{M, W}\left(\left\{v_{i, j}^{k}\left(v_{k}, \phi_{k}\right)\right\}_{i, j, k},\left\{v_{l}^{p+1}(\xi, \eta)\right\}_{l}\right) .
\end{aligned}
$$

We choose as our approximate solution the unique $\left\{\left\{z_{i, j}^{k}\left(v_{k}, \phi_{k}\right)\right\}_{i, j, k},\left\{z_{l}^{p+1}(\xi, \eta)\right\}_{l}\right\} \in$ $\Pi^{M, W}$, the space of spectral element functions, which minimizes the functional $\mathfrak{r}^{M, W}\left(\left\{v_{i, j}^{k}\left(v_{k}, \phi_{k}\right)\right\}_{i, j, k},\left\{v_{l}^{p+1}(\xi, \eta)\right\}_{l}\right)$ over all $\left\{\left\{v_{i, j}^{k}\left(v_{k}, \phi_{k}\right)\right\}_{i, j, k},\left\{v_{l}^{p+1}(\xi, \eta)\right\}_{l}\right\}$.

A brief description of the solution procedure is now given; a more detailed examination is provided in $\S 6$. The above method is essentially a least-squares method and the solution can be obtained by using preconditioned conjugate gradient techniques (PCGM) to solve the normal equations. To be able to do so we must be able to compute the residuals in the normal equations inexpensively. In [5]14] it has been shown how to compute these efficiently on a distributed memory parallel computer, without having to filter the coefficients of the differential operator and the data. The evaluation of the residuals on each element requires the interchange of boundary values between neighbouring elements.

The values of the spectral element functions at the vertices of the polygonal domain constitute the set of common boundary values $U_{B}$. Since the dimension of the set of common boundary values is so small a nearly exact approximation to the Schur Complement matrix can be computed. Now on the subspace of spectral element functions which vanish at the set of common boundary values it is possible to define a preconditioner for the matrix in the normal equations such that the condition number of the preconditioned system is $O\left((\ln W)^{2}\right)$. Moreover, the preconditioner is a block diagonal matrix such that each diagonal block corresponds to a different element, and so can be easily inverted.

Hence an exponentially accurate approximation $\mathbb{S}^{a}$ to the Schur Complement matrix $\mathbb{S}$ can be computed using $O(W \ln W)$ iterations of the PCGM. To solve the normal equations the residual in the equations for the Schur Complement $\mathbb{S} U_{B}=h_{B}$ must be computed to exponential accuracy and this can be done using $O(W \ln W)$ iterations of the PCGM. The common boundary values $U_{B}$ are then given by $U_{B}=\left(\mathbb{S}^{a}\right)^{-1} h_{B}$. The remaining values can then be obtained using $O(W \ln W)$ iterations of the PCGM.

\section{Error estimates}

Let $\left\{\left\{z_{i, j}^{k}\left(v_{k}, \phi_{k}\right)\right\}_{i, j, k},\left\{z_{l}^{p+1}(\xi, \eta)\right\}_{l}\right\}$ minimize $\mathfrak{r}^{M, W}\left(\left\{v_{i, j}^{k}\left(v_{k}, \phi_{k}\right)\right\}_{i, j, k},\left\{v_{l}^{p+1}(\xi, \eta)\right\}_{l}\right)$ over all $\left\{\left\{v_{i, j}^{k}\left(v_{k}, \phi_{k}\right)\right\}_{i, j, k},\left\{v_{l}^{p+1}(\xi, \eta)\right\}_{l}\right\} \in \Pi^{M, W}$, the space of spectral element functions. Here $z_{i, 1}^{k}=b_{k}$ for all $i, z_{i, j}^{k}\left(v_{k}, \phi_{k}\right)$ is a polynomial in $v_{k}$ and $\phi_{k}$ of degree $W_{j}$, $W_{j} \leq W$ and $z_{l}^{p+1}(\xi, \eta)$ is a polynomial in $\xi$ and $\eta$ of degree $W$ as defined in $\S 3$. We choose $W$ proportional to $M$. Then we have the following error estimate.

Theorem 5.1. Let $a_{k}=u\left(A_{k}\right)$. Let $U_{i, j}^{k}\left(v_{k}, \phi_{k}\right)=u\left(v_{k}, \phi_{k}\right)$ for $\left(v_{k}, \phi_{k}\right) \in \hat{\Omega}_{i, j}^{k}$ and $U_{l}^{p+1}(\xi, \eta)=u(\xi, \eta)$ for $(\xi, \eta) \in S$. Let $\alpha j \leq W_{j} \leq W$ for some positive $\alpha$ for $j>2$. Then there exists positive constants $C$ and $b$ such that for $W$ large enough the estimate

$$
\sum_{k=1}^{p}\left|b_{k}-a_{k}\right|^{2}+\sum_{k=1}^{p} \sum_{j=2}^{M} \sum_{i=1}^{I_{k}}\left(\rho \mu_{k}^{M+1-j}\right)^{-2 \lambda_{k}}
$$




$$
\begin{aligned}
& \times\left\|\left(z_{i, j}^{k}-U_{i, j}^{k}\right)\left(v_{k}, \phi_{k}\right)-\left(b_{k}-a_{k}\right)\right\|_{2, \hat{\Omega}_{i, j}^{k}}^{2} \\
& +\sum_{l=1}^{L}\left\|\left(z_{l}^{p+1}-U_{l}^{p+1}\right)(\xi, \eta)\right\|_{2, S}^{2} \leq C \mathrm{e}^{-b W}
\end{aligned}
$$

holds.

We use the differentiability estimates stated in Proposition 2.1 to prove the result. The proof of the above Theorem is very similar to the proof of Theorem 3.1 in [14] and hence is omitted.

Remark. We can construct a set of corrections $\left\{\left\{c_{i, j}^{k}\left(v_{k}, \phi_{k}\right)\right\}_{i, j, k},\left\{c_{l}^{p+1}(\xi, \eta)\right\}_{l}\right\} \in \Pi^{M, W}$ the set of spectral element functions, so that corrected solution $\left\{\left\{\hat{z}_{i, j}^{k}\left(v_{k}, \phi_{k}\right)\right\}_{i, j, k}\right.$, $\left.\left\{\hat{z}_{l}^{p+1}(\xi, \eta)\right\}_{l}\right\}$ defined by

$$
\begin{aligned}
& \left\{\left\{\hat{z}_{i, j}^{k}\left(v_{k}, \phi_{k}\right)\right\}_{i, j, k},\left\{\hat{z}_{l}^{p+1}(\xi, \eta)\right\}_{l}\right\} \\
& \quad=\left\{\left\{z_{i, j}^{k}\left(v_{k}, \phi_{k}\right)\right\}_{i, j, k},\left\{z_{l}^{p+1}(\xi, \eta)\right\}_{l}\right\}+\left\{\left\{c_{i, j}^{k}\left(v_{k}, \phi_{k}\right)\right\}_{i, j, k},\left\{c_{l}^{p+1}(\xi, \eta)\right\}_{l}\right\}
\end{aligned}
$$

is conforming and belongs to $H^{1}(\Omega)$. These corrections are defined in $\S 3.5$ of [14]. Then the error estimate

$$
\|(u-\hat{z})(x, y)\|_{1, \Omega} \leq C \mathrm{e}^{-b W}
$$

holds for $W$ large enough. Here $C$ and $b$ denote constants. These constructions are similar to Lemma 4.57 in [12].

\section{Parallelization and preconditioning}

Let $U$ be a vector assembled from $\left\{g_{k}\right\}_{k=1}^{p}$, where $u_{i, 1}^{k}=g_{k}$ for all $i$, and the values of $\left\{\left\{u_{i, j}^{k}\left(v_{k}, \phi_{k}\right)\right\}_{i, j, k},\left\{u_{l}^{p+1}(\xi, \eta)\right\}_{l}\right\}$ at the Gauss-Lobatto-Legendre points are arranged in lexicographic order for $1 \leq k \leq p, 2 \leq j \leq J_{k}, 1 \leq i \leq I_{k, j}$. Let $\left\{\left\{z_{i, j}^{k}\left(v_{k}, \phi_{k}\right)\right\}_{i, j, k},\left\{z_{l}^{p+1}(\xi, \eta)\right\}_{l}\right\}$ minimize $\mathfrak{r}^{M, W}\left(\left\{v_{i, j}^{k}\left(v_{k}, \phi_{k}\right)\right\}_{i, j, k},\left\{v_{l}^{p+1}(\xi, \eta)\right\}_{l}\right)$ over all $\left\{\left\{v_{i, j}^{k}\left(v_{k}, \phi_{k}\right)\right\}_{i, j, k},\left\{v_{l}^{p+1}(\xi, \eta)\right\}_{l}\right\} \in \Pi^{M, W}$, the space of spectral element functions.

Let $U_{B}$ denote the values $\left\{g_{k}\right\}_{k=1}^{p}$ and $U_{I}$ the remaining values of $U$. We now define a quadratic form

$$
\begin{aligned}
\mathscr{Z}^{M, W} & \left(\left\{u_{i, j}^{k}\left(v_{k}, \phi_{k}\right)\right\}_{i, j, k},\left\{u_{l}^{p+1}(\xi, \eta)\right\}_{l}\right) \\
= & \sum_{k=1}^{p}\left|g_{k}\right|^{2}+\sum_{k=1}^{p} \sum_{j=2}^{M} \sum_{i=1}^{I_{k}}\left(\rho \mu_{k}^{M+1-j}\right)^{-2 \lambda_{k}}\left\|u_{i, j}^{k}(\xi, \eta)-g_{k}\right\|_{2, S}^{2} \\
& +\sum_{l=1}^{L}\left\|u_{l}^{p+1}(\xi, \eta)\right\|_{2, S}^{2} .
\end{aligned}
$$

It should be noted that $u_{i, 1}^{k}\left(v_{k}, \phi_{k}\right)=g_{k}$ for $1 \leq i \leq I_{k}$. Moreover for $j \leq M$, $\xi$ is a linear function of $v_{k}$ and $\eta$ is a linear function of $\phi_{k}$ such that the linear mapping $M_{i, j}^{k}(\xi, \eta)$ maps the master square $S$ onto $\hat{\Omega}_{i, j}^{k}$. 
To solve the minimization problem we have to solve a system of equations of the form

$$
A Z=h .
$$

Here $A$ is a symmetric positive definite matrix and

$$
\mathscr{V}^{M, W}\left(\left\{u_{i, j}^{k}\left(v_{k}, \phi_{k}\right)\right\}_{i, j, k},\left\{u_{l}^{p+1}(\xi, \eta)\right\}_{l}\right)=U^{T} A U,
$$

where $\mathscr{V}^{M, W}\left(\left\{u_{i, j}^{k}\left(v_{k}, \phi_{k}\right)\right\}_{i, j, k},\left\{u_{l}^{p+1}(\xi, \eta)\right\}_{l}\right)$ is as defined in (3.15) in $\S 3$.

Now $A$ has the form

$$
A=\left[\begin{array}{ll}
A_{I I} & A_{I B} \\
A_{B I} & A_{B B}
\end{array}\right]
$$

corresponding to the decomposition of $U$ as

$$
U=\left[\begin{array}{c}
U_{I} \\
U_{B}
\end{array}\right]
$$

and $h$ has the form

$$
h=\left[\begin{array}{l}
h_{I} \\
h_{B}
\end{array}\right] .
$$

To solve the matrix equation (6.2) we use the block L-U factorization of $A$, viz.

$$
A=\left[\begin{array}{cc}
I & 0 \\
A_{I B}^{T} A_{I I}^{-1} & I
\end{array}\right]\left[\begin{array}{cc}
A_{I I} & 0 \\
0 & \mathbb{S}
\end{array}\right]\left[\begin{array}{cc}
I & A_{I I}^{-1} A_{I B} \\
0 & I
\end{array}\right],
$$

where the Schur Complement matrix $\mathbb{S}$ is defined as

$$
\mathbb{S}=A_{B B}-A_{I B}^{T} A_{I I}^{-1} A_{I B} .
$$

To solve the matrix equation (6.2) based on the L-U factorization of $A$ given in (6.5) reduces to solving the system of equations

$$
\mathbb{S} Z_{B}=\tilde{h}_{B}
$$

where

$$
\tilde{h}_{B}=h_{B}-A_{I B}^{T} A_{I I}^{-1} h_{I}
$$

The feasibility of such a process depends on our being able to compute $A_{I B} V_{B}, A_{I I} V_{I}$ and $A_{B B} V_{B}$ for any $V_{I}, V_{B}$ efficiently and this can always be done since $A V$ can be computed inexpensively as explained in ch. 3 of [14].

However in addition to this it is imperative that we should be able to construct effective preconditioners for the matrix $A_{I I}$ so that the condition number of the preconditioned system is as small as possible. If this can be done then it will be possible to compute $A_{I I}^{-1} V_{I}$ efficiently using the preconditioned conjugate gradient method (PCGM) for any vector $V_{I}$.

Consider the space of spectral element functions $\Pi_{0}^{M, W}$, such that for $\left\{\left\{u_{i, j}^{k}\left(v_{k}, \phi_{k}\right)\right\}_{i, j, k}\right.$, $\left.\left\{u_{l}^{p+1}(\xi, \eta)\right\}_{l}\right\} \in \Pi_{0}^{M, W}$ we have $u_{i, 1}^{k}=0$ for all $i$ and $k$. Let $U$ be the vector corresponding 
to the spectral element function $\left\{\left\{u_{i, j}^{k}\left(v_{k}, \phi_{k}\right)\right\}_{i, j, k},\left\{u_{l}^{p+1}(\xi, \eta)\right\}_{l}\right\}$. Then $U_{B}=0$ and $U=$ $\left[\begin{array}{c}U_{I} \\ 0\end{array}\right]$ and so

$$
\mathscr{V}^{M, W}\left(\left\{u_{i, j}^{k}\left(v_{k}, \phi_{k}\right)\right\}_{i, j, k},\left\{u_{l}^{p+1}(\xi, \eta)\right\}_{l}\right)=U_{I}^{T} A_{I I} U_{I}
$$

Now using Theorem 3.1 we have the following result.

Let $\left\{\left\{u_{i, j}^{k}\left(v_{k}, \phi_{k}\right)\right\}_{i, j, k},\left\{u_{l}^{p+1}(\xi, \eta)\right\}_{l}\right\} \in \Pi_{0}^{M, W}$. Then the estimate

$$
\begin{gathered}
\sum_{k=1}^{p} \sum_{j=2}^{M} \sum_{i=1}^{I_{k}}\left(\rho \mu_{k}^{M+1-j}\right)^{-2 \lambda_{k}}\left\|u_{i, j}^{k}(\xi, \eta)\right\|_{2, S}^{2}+\sum_{l=1}^{L}\left\|u_{l}^{p+1}(\xi, \eta)\right\|_{2, S}^{2} \\
\leq C(\ln W)^{2} \mathscr{V}^{M, W}\left(\left\{u_{i, j}^{k}\left(v_{k}, \phi_{k}\right)\right\}_{i, j, k},\left\{u_{l}^{p+1}(\xi, \eta)\right\}_{l}\right)
\end{gathered}
$$

holds for $W$ large enough. In the above, $u_{i, 1}^{k}=0$ for $1 \leq k \leq p$ and $1 \leq i \leq I_{k}$.

Let us define the quadratic form

$$
\begin{aligned}
\mathscr{U}^{M, W}\left(\left\{u_{i, j}^{k}\left(v_{k}, \phi_{k}\right)\right\}_{i, j, k},\left\{u_{l}^{p+1}(\xi, \eta)\right\}_{l}\right) \\
=\sum_{k=1}^{p} \sum_{j=2}^{M} \sum_{i=1}^{I_{k}}\left(\rho \mu_{k}^{M+1-j}\right)^{-2 \lambda_{k}}\left\|u_{i, j}^{k}(\xi, \eta)\right\|_{2, S}^{2}+\sum_{l=1}^{L}\left\|u_{l}^{p+1}(\xi, \eta)\right\|_{2, S}^{2}
\end{aligned}
$$

for all $\left\{\left\{u_{i, j}^{k}\left(v_{k}, \phi_{k}\right)\right\}_{i, j, k},\left\{u_{l}^{p+1}(\xi, \eta)\right\}_{l}\right\} \in \Pi_{0}^{M, W}$.

Now using the trace theorems for Sobolev spaces it can be concluded that there exists a constant $K$ such that

$$
\begin{aligned}
\mathscr{V}^{M, W} & \left(\left\{u_{i, j}^{k}\left(v_{k}, \phi_{k}\right)\right\}_{i, j, k},\left\{u_{l}^{p+1}(\xi, \eta)\right\}_{l}\right) \\
\leq & K \mathscr{U}^{M, W}\left(\left\{u_{i, j}^{k}\left(v_{k}, \phi_{k}\right)\right\}_{i, j, k},\left\{u_{l}^{p+1}(\xi, \eta)\right\}_{l}\right)
\end{aligned}
$$

for $\left\{\left\{u_{i, j}^{k}\left(v_{k}, \phi_{k}\right)\right\}_{i, j, k},\left\{u_{l}^{p+1}(\xi, \eta)\right\}_{l}\right\} \in \Pi_{0}^{M, W}$.

Hence using (6.10) and (6.12) it follows that there exists a constant $C$ such that

$$
\begin{aligned}
\frac{1}{C} \mathscr{V}^{M, W}\left(\left\{u_{i, j}^{k}\left(v_{k}, \phi_{k}\right)\right\}_{i, j, k},\left\{u_{l}^{p+1}(\xi, \eta)\right\}_{l}\right) \\
\quad \leq \mathscr{U}^{M, W}\left(\left\{u_{i, j}^{k}\left(v_{k}, \phi_{k}\right)\right\}_{i, j, k},\left\{u_{l}^{p+1}(\xi, \eta)\right\}_{l}\right) \\
\quad \leq C(\ln W)^{2} \mathscr{V}^{M, W}\left(\left\{u_{i, j}^{k}\left(v_{k}, \phi_{k}\right)\right\}_{i, j, k},\left\{u_{l}^{p+1}(\xi, \eta)\right\}_{l}\right)
\end{aligned}
$$

for all $\left\{\left\{u_{i, j}^{k}\left(v_{k}, \phi_{k}\right)\right\}_{i, j, k},\left\{u_{l}^{p+1}(\xi, \eta)\right\}_{l}\right\} \in \Pi_{0}^{M, W}$.

Thus the two forms $\mathscr{V}^{M, W}\left(\left\{u_{i, j}^{k}\left(v_{k}, \phi_{k}\right)\right\}_{i, j, k},\left\{u_{l}^{p+1}(\xi, \eta)\right\}_{l}\right)$ and $\mathscr{U}^{M, W}\left(\left\{u_{i, j}^{k}\left(v_{k}, \phi_{k}\right)\right\}_{i, j, k}\right.$, $\left.\left\{u_{l}^{p+1}(\xi, \eta)\right\}_{l}\right)$ are spectrally equivalent.

We can now use the quadratic form $\mathscr{U}^{M, W}\left(\left\{u_{i, j}^{k}\left(v_{k}, \phi_{k}\right)\right\}_{i, j, k},\left\{u_{l}^{p+1}(\xi, \eta)\right\}_{l}\right)$ which consists of a decoupled set of quadratic forms on each element as a preconditioner for $A_{I I}$. This can be done by inverting the block diagonal matrix representation for $\mathscr{U}^{M, W}\left(\left\{u_{i, j}^{k}\left(v_{k}, \phi_{k}\right)\right\}_{i, j, k},\left\{u_{l}^{p+1}(\xi, \eta)\right\}_{l}\right)$. 
Now from (6.13) we can conclude that if we were to compute $\left(A_{I I}\right)^{-1} U_{I}$ using the PCGM then the condition number of the preconditioned matrix would be $O\left((\ln W)^{2}\right)$. Hence, to compute $\left(A_{I I}\right)^{-1} U_{I}$ to an accuracy of $O\left(\mathrm{e}^{-b W}\right)$ would require $O(W \ln W)$ iterations of the PCGM.

We now return to the steps involved in solving the system of equations (6.2). As a first step it would be necessary to solve the much smaller system of equations (6.7). Here the dimension of the vector $Z_{B}$ is $p$, the number of vertices of the domain $\Omega$. Now to be able to solve (6.7) to an accuracy of $O\left(\mathrm{e}^{-b W}\right)$ using PCGM the residual

$$
R_{B}=\mathbb{S} U_{B}-\tilde{h}_{B}
$$

needs to be computed with the same accuracy and in an efficient manner. The bottleneck in computing $R_{B}$ consists in computing $\left(A_{I I}\right)^{-1} A_{I B} U_{B}$ to an accuracy of $O\left(\mathrm{e}^{-b W}\right)$ and it has already been seen that this can be done using $O(W \ln W)$ iterations of the PCGM for computing $\left(A_{I I}\right)^{-1} A_{I B} U_{B}$ for a given vector $U_{B}$.

We now show that it is possible to explicitly construct the Schur Complement matrix $\mathbb{S}$ in $O(W \ln W)$ iterations of the PCGM. $\mathbb{S}$ is a $p \times p$ matrix. Let $e_{k}$ be a column vector of dimension $p$ with 1 in the $k$ th place and 0 elsewhere. Let $\mathbb{S}_{k}=\mathbb{S} e_{k}$.

Then the Schur Complement matrix $\mathbb{S}$ can be written as

$$
\mathbb{S}=\left[\mathbb{S}_{1}, \mathbb{S}_{2}, \ldots, \mathbb{S}_{p}\right]
$$

Now by a well known result on the Schur Complement we have

$$
\begin{aligned}
U_{B}^{T} \mathbb{S} U_{B} & =\min _{V: v_{B}=U_{B}} V^{T} A V \\
& =\min _{v_{i, j}^{k}: v_{i, 1}^{k}=g_{k}} \mathscr{V}^{M, W}\left(\left\{v_{i, j}^{k}\left(v_{k}, \phi_{k}\right)\right\}_{i, j, k},\left\{v_{l}^{p+1}(\xi, \eta)\right\}_{l}\right) .
\end{aligned}
$$

Here $U_{B}=\left[g_{1}, g_{2}, \ldots, g_{p}\right]^{T}$. Hence using Theorem 3.1 we conclude that

$$
U_{B}^{T} \mathbb{S} U_{B} \geq \frac{C}{(\ln W)^{2}}\left\|U_{B}\right\|^{2} .
$$

And so we obtain

$$
\left\|\mathbb{S}^{-1}\right\| \leq C(\ln W)^{2} .
$$

Here the norm denoted is the matrix norm induced by the Euclidean norm. Now

$$
\mathbb{S}_{k}=\mathbb{S e}_{k}=\left(A_{B B}-A_{I B}^{T} A_{I I}^{-1} A_{I B}\right) \mathrm{e}_{k} .
$$

Let $\left(\mathbb{S}_{k}\right)^{a}$ be the approximation to $\mathbb{S}_{k}$ computed using $O(W \ln W)$ iterations of the PCGM to compute $A_{I I}^{-1} A_{I B} e_{k}$. Then

$$
\left\|\mathbb{S}_{k}-\mathbb{S}_{k}^{a}\right\|=O\left(\mathrm{e}^{-b W}\right) .
$$

Let $\mathbb{S}^{a}$ denote the matrix

$$
\mathbb{S}^{a}=\left[\mathbb{S}_{1}^{a}, \mathbb{S}_{2}^{a}, \ldots, \mathbb{S}_{p}^{a}\right]
$$


Clearly

$$
\left\|\mathbb{S}-\mathbb{S}^{a}\right\|=O\left(\mathrm{e}^{-b W}\right) .
$$

Now to compute $\mathbb{S}^{a}$ requires $O(W \ln W)$ iterations of the PCGM since $p$ is a fixed constant. Hence we can solve (6.7) as

$$
\mathbb{S Z}_{B}=\tilde{h}_{B}
$$

by replacing $\mathbb{S}$ by the matrix $\mathbb{S}^{a}$. Let $Z_{B}^{a}$ be the solution of

$$
\mathbb{S}^{a} Z_{B}^{a}=\tilde{h}_{B}
$$

Since

$$
\mathbb{S}^{a}=\mathbb{S}+\delta \mathbb{S}
$$

we have

$$
\left(\mathbb{S}^{a}\right)^{-1}=\left(I+\mathbb{S}^{-1} \delta \mathbb{S}\right) \mathbb{S}^{-1}
$$

Thus

$$
\left\|\mathbb{S}^{-1}-\left(\mathbb{S}^{a}\right)^{-1}\right\| \leq 2\left\|\mathbb{S}^{-1}\right\|^{2}\|\delta \mathbb{S}\| \leq O\left((\ln W)^{4}\right)\|\delta \mathbb{S}\|
$$

for $\|\delta \mathbb{S}\|$ small enough.

Hence

$$
\left\|\mathbb{S}^{-1}-\left(\mathbb{S}^{a}\right)^{-1}\right\|=O\left(\mathrm{e}^{-b W}\right) .
$$

Therefore

$$
\left\|Z_{B}^{a}-Z_{B}\right\|=O\left(\mathrm{e}^{-b W}\right) .
$$

Having solved for $Z_{B}$ we obtain $Z_{I}$ by solving

$$
A_{I I} Z_{I}=h_{I}-A_{I B} Z_{B}
$$

using $O(W \ln W)$ iterations of the PCGM. Hence the solution $Z$ can be obtained to exponential accuracy using $O(W \ln W)$ iterations of the PCGM.

We shall now briefly examine the complexity of the solution procedure for the $h-p$ finite element method. Since finite elements have to be continuous along the sides of the elements, the cardinality of the set of common boundary value is large in the h-p finite element method. Let $\mathbb{S}$ denote the Schur Complement matrix for the h-p finite element method. In [1]10] it has been shown that an approximation $\mathbb{S}^{a}$ to $\mathbb{S}$ can be obtained such that the condition number $\chi$ of the preconditioned system satisfies

$$
\chi \leq C\left(1+(\ln W)^{2}\right),
$$

where $C$ denotes a constant. Then to solve $\mathbb{S} U_{B}=h_{B}$ to an accuracy $O\left(\mathrm{e}^{-b W}\right)$ will require $O(W \ln W)$ iterations of the PCGM using $\mathbb{S}^{a}$ as a preconditioner. Now to compute the residual in the Schur Complement system to an accuracy of $O\left(\mathrm{e}^{-b W}\right)$ requires $O(W)$ iterations of the PCGM to compute $A_{I I}^{-1} A_{I B} V_{B}$. Hence we would need to perform $O\left(W^{2} \ln W\right)$ iterations of the PCGM for computing $A_{I I}^{-1} V_{I}$, where $V_{I}$ will vary after every sequence of $O(W \ln W)$ steps. So the h-p finite element method requires $O\left(W^{2} \ln W\right)$ iterations of the PCGM to obtain the solution.

Hence the proposed method is faster than h-p finite element method by a factor of $O(W)$. 


\section{Technical results}

Lemma 7.1. Let $\left\{\left\{u_{i, j}^{k}\left(v_{k}, \phi_{k}\right)\right\}_{i, j, k},\left\{u_{l}^{p+1}(\xi, \eta)\right\}_{l}\right\} \in \Pi^{M, W}$. Then there exists $\left\{\left\{v_{i, j}^{k}\right.\right.$ $\left.\left.\left(v_{k}, \phi_{k}\right)\right\}_{i, j, k},\left\{v_{l}^{p+1}(\xi, \eta)\right\}_{l}\right\}$ such that $v_{i, 1}^{k}=0$ for all $i, k, v_{i, j}^{k} \in H^{2}\left(\hat{\Omega}_{i, j}^{k}\right)$ for $2 \leq j \leq M$ and all $i$ and $k, v_{l}^{p+1} \in H^{2}(S)$ for $l=1,2, \ldots, L$ and $w=u+v \in H_{\beta}^{2,2}(\Omega)$. Moreover the estimate

$$
\begin{aligned}
& \sum_{k=1}^{p} \sum_{i=1}^{I_{k}} \sum_{j=2}^{M}\left(\rho \mu_{k}^{M+1-j}\right)^{-2 \lambda_{k}}\left\|v_{i, j}^{k}\left(v_{k}, \phi_{k}\right)\right\|_{2, \hat{\Omega}_{i, j}^{k}}^{2}+\sum_{l=1}^{L}\left\|v_{l}^{p+1}(\xi, \eta)\right\|_{2, S}^{2} \\
& \leq C(\ln W)^{2}\left(\sum_{k=1}^{p} \sum_{\gamma_{s} \subseteq \Omega^{k} \cup B_{\rho}^{k}, \mu\left(\hat{\gamma}_{s}\right)<\infty} d\left(A_{k}, \gamma_{s}\right)^{-2 \lambda_{k}}\right. \\
& \times\left(\left\|\left[u^{k}\right]\right\|_{0, \hat{\gamma}_{s}}^{2}+\left\|\left[\left(u_{v_{k}}^{k}\right)^{a}\right]\right\|_{1 / 2, \hat{\gamma}_{s}}^{2}+\left\|\left[\left(u_{\phi_{k}}^{k}\right)^{a}\right]\right\|_{1 / 2, \hat{\gamma}_{s}}^{2}\right) \\
&\left.+\sum_{\gamma_{s} \subseteq \Omega^{p+1}}\left(\left\|\left[u^{p+1}\right]\right\|_{0, \gamma_{s}}^{2}+\left\|\left[\left(u_{x_{1}}^{p+1}\right)^{a}\right]\right\|_{1 / 2, \gamma_{s}}^{2}+\left\|\left[\left(u_{x_{2}}^{p+1}\right)^{a}\right]\right\|_{1 / 2, \gamma_{s}}^{2}\right)\right) \\
&+\varepsilon_{W}\left(\sum_{k=1}^{p} \sum_{i=1}^{I_{k}} \sum_{j=2}^{M}\left(\rho \mu_{k}^{M+1-j}\right)^{-2 \lambda_{k}}\left\|u_{i, j}^{k}\left(v_{k}, \phi_{k}\right)-h_{k}\right\|_{2, \hat{\Omega}_{i, j}^{k}}^{2}\right. \\
&\left.+\sum_{l=1}^{L}\left\|u_{l}^{p+1}(\xi, \eta)\right\|_{2, S}^{2}\right)
\end{aligned}
$$

holds. Here $\varepsilon_{W}$ is exponentially small in $W$.

We first make a correction $\left\{\left\{r_{i, j}^{k}\left(v_{k}, \phi_{k}\right)\right\}_{i, j, k},\left\{r_{l}^{p+1}(\xi, \eta)\right\}_{l}\right\}$ such that $r_{i, 1}^{k}=0$ for all $i$ and $k$ and at the vertices $\hat{Q}_{l}$ for $l=1, \ldots, 4$ of $\hat{\Omega}_{i, j}^{k}$,

$$
\begin{aligned}
\left(u_{i, j}^{k}+r_{i, j}^{k}\right)\left(\hat{Q}_{l}\right) & =\bar{u}\left(\hat{Q}_{l}\right), \\
\left(\left(u_{i, j}^{k}\right)_{v_{k}}+\left(r_{i, j}^{k}\right)_{v_{k}}\right)\left(\hat{Q}_{l}\right) & =\bar{u}_{v_{k}}\left(\hat{Q}_{l}\right), \\
\left(\left(u_{i, j}^{k}\right)_{\phi_{k}}+\left(r_{i, j}^{k}\right)_{\phi_{k}}\right)\left(\hat{Q}_{l}\right) & =\bar{u}_{\phi_{k}}\left(\hat{Q}_{l}\right),
\end{aligned}
$$

provided $Q_{l}$ is not a vertex of $\Omega_{i, 1}^{k}$ for all $i, k$. If $Q_{l}$ is a vertex of $\Omega_{i, 1}^{k}$ choose $r_{i, 2}^{k}$ such that

$$
\begin{aligned}
\left(u_{i, 2}^{k}+r_{i, 2}^{k}\right)\left(\hat{Q}_{l}\right) & =u_{i, 1}^{k}\left(\hat{Q}_{l}\right), \\
\left(\left(u_{i, 2}^{k}\right)_{v_{k}}+\left(r_{i, 2}^{k}\right)_{v_{k}}\right)\left(\hat{Q}_{l}\right) & =\left(u_{i, 1}^{k}\right)_{v_{k}}\left(\hat{Q}_{l}\right), \\
\left(\left(u_{i, 2}^{k}\right)_{\phi_{k}}+\left(r_{i, 2}^{k}\right)_{\phi_{k}}\right)\left(\hat{Q}_{l}\right) & =\left(u_{i, 1}^{k}\right)_{\phi_{k}}\left(\hat{Q}_{l}\right) .
\end{aligned}
$$

Here $\bar{s}\left(\hat{Q}_{l}\right)$ denotes the average of the values of $s$ at $\hat{Q}_{l}$ over all the elements which have $\hat{Q}_{l}$ as a vertex.

We can find a polynomial $r_{i, j}^{k}\left(v_{k}, \phi_{k}\right)$ on $\hat{\Omega}_{i, j}^{k}$ such that $r_{i, j}^{k}\left(\hat{Q}_{l}\right)=a_{l},\left(r_{i, j}^{k}\right) v_{k}\left(\hat{Q}_{l}\right)=$ $b_{l},\left(r_{i, j}^{k}\right)_{\phi_{k}}\left(\hat{Q}_{l}\right)=c_{l}$ for $l=1, \ldots, 4$. Here the values $a_{l}, b_{l}, c_{l}$ are defined by (7.2). Moreover $r_{i, j}^{k}$ is a polynomial of degree less than or equal to four and the estimate 


$$
\left\|r_{i, j}^{k}\left(v_{k}, \phi_{k}\right)\right\|_{2, \hat{\Omega}_{i, j}^{k}}^{2} \leq C\left(\sum_{l=1}^{4}\left|a_{l}\right|^{2}+\left|b_{l}\right|^{2}+\left|c_{l}\right|^{2}\right)
$$

holds for $j \geq 2$ and all $i$ and $k$. Next consider $\Omega_{l}^{p+1} \in \Omega^{p+1}$. Now

$$
\begin{aligned}
& \left(u_{l}^{p+1}\right)_{x_{1}}=\left(u_{l}^{p+1}\right)_{\xi} \xi_{x_{1}}+\left(u_{l}^{p+1}\right)_{\eta} \eta_{x_{1}}, \text { and } \\
& \left(u_{l}^{p+1}\right)_{x_{2}}=\left(u_{l}^{p+1}\right)_{\xi} \xi_{x_{2}}+\left(u_{l}^{p+1}\right)_{\eta} \eta_{x_{2}} .
\end{aligned}
$$

Let $\hat{\xi}_{x_{1}}, \hat{\xi}_{x_{2}}, \hat{\eta}_{x_{1}}$ and $\hat{\eta}_{x_{2}}$ denote the polynomials of degree $W$ in $\xi$ and $\eta$ separately which are the approximations to $\xi_{x_{1}}, \xi_{x_{2}}, \eta_{x_{1}}$ and $\eta_{x_{2}}$ in the space of polynomial of degree $W$ as defined in Theorem 4.46 of [12].

Let $P_{j}, j=1, \ldots, 4$ denote the vertices of $S$. Then $\hat{\xi}_{x_{i}}\left(P_{j}\right)=\xi_{x_{i}}\left(P_{j}\right)$ and $\hat{\eta}_{x_{i}}\left(P_{j}\right)=\eta_{x_{i}}\left(P_{j}\right)$ for $i=1,2$ and $j=1, \ldots, 4$. Now

$$
\begin{aligned}
& \left(u_{l}^{p+1}\right)_{x_{1}}^{a}=\left(u_{l}^{p+1}\right)_{\xi} \hat{\xi}_{x_{1}}+\left(u_{l}^{p+1}\right)_{\eta} \hat{\eta}_{x_{1}}, \text { and } \\
& \left(u_{l}^{p+1}\right)_{x_{2}}^{a}=\left(u_{l}^{p+1}\right)_{\xi} \hat{\xi}_{x_{2}}+\left(u_{l}^{p+1}\right)_{\eta} \hat{\eta}_{x_{2}} .
\end{aligned}
$$

Hence $\left(u_{l}^{p+1}\right)_{x_{i}}^{a}\left(P_{j}\right)=\left(u_{l}^{p+1}\right)_{x_{i}}\left(P_{j}\right)$, for $i=1,2$ and $j=1, \ldots, 4$. Therefore we can find a polynomial $r_{l}^{p+1}(\xi, \eta)$ on $S=\left(M_{l}^{p+1}\right)^{-1}\left(\Omega_{l}^{p+1}\right)$ such that for $j=1, \ldots, 4$,

$$
\begin{aligned}
& \left(u_{l}^{p+1}+r_{l}^{p+1}\right)\left(P_{j}\right)=\bar{u}\left(P_{j}\right), \\
& \left(\left(u_{l}^{p+1}\right)_{x_{1}}+\left(r_{l}^{p+1}\right)_{x_{1}}\right)\left(P_{j}\right)=\bar{u}_{x_{1}}\left(P_{j}\right), \text { and } \\
& \left(\left(u_{l}^{p+1}\right)_{x_{2}}+\left(r_{l}^{p+1}\right)_{x_{2}}\right)\left(P_{j}\right)=\bar{u}_{x_{2}}\left(P_{j}\right) .
\end{aligned}
$$

Now let $q(y)$ be a polynomial of degree $W$ defined on $I=(-1,1)$. Then by Theorem 4.79 of [12]

$$
\|q\|_{L^{\infty}(\bar{I})}^{2} \leq C(\ln W)\|q\|_{1 / 2, I}^{2}
$$

Here $C$ is a constant. Hence using (7.3) and (7.4) we obtain

$$
\begin{aligned}
& \sum_{k=1}^{p} \sum_{i=1}^{I_{k}} \sum_{j=2}^{M}\left(\rho \mu_{k}^{M+1-j}\right)^{-2 \lambda_{k}}\left\|r_{i, j}^{k}\left(v_{k}, \phi_{k}\right)\right\|_{2, \hat{\Omega}_{i, j}^{k}}^{2}+\sum_{l=1}^{L}\left\|r_{l}^{p+1}(\xi, \eta)\right\|_{2, S}^{2} \\
& \leq K(\ln W)\left(\sum_{k=1}^{p} \sum_{\gamma_{s} \subseteq \Omega^{k} \cup B_{\rho}^{k}, \mu\left(\hat{\gamma}_{s}\right)<\infty} d\left(A_{k}, \gamma_{s}\right)^{-2 \lambda_{k}}\right. \\
& \quad \times\left(\left\|\left[u^{k}\right]\right\|_{0, \hat{\gamma}_{s}}^{2}+\left\|\left[\left(u_{v_{k}}^{k}\right)^{a}\right]\right\|_{1 / 2, \hat{\gamma}_{s}}^{2}+\left\|\left[\left(u_{\phi_{k}}^{k}\right)^{a}\right]\right\|_{1 / 2, \hat{\gamma}_{s}}^{2}\right) \\
&\left.\quad+\sum_{\gamma_{s} \subseteq \Omega^{p+1}}\left(\left\|\left[u^{p+1}\right]\right\|_{0, \gamma_{s}}^{2}+\left\|\left[\left(u_{x_{1}}^{p+1}\right)^{a}\right]\right\|_{1 / 2, \gamma_{s}}^{2}+\left\|\left[\left(u_{x_{2}}^{p+1}\right)^{a}\right]\right\|_{1 / 2, \gamma_{s}}^{2}\right)\right)
\end{aligned}
$$




$$
\begin{aligned}
& +\varepsilon_{W}\left(\sum_{k=1}^{p} \sum_{i=1}^{I_{k}} \sum_{j=2}^{M}\left(\rho \mu_{k}^{M+1-j}\right)^{-2 \lambda_{k}}\left\|u_{i, j}^{k}\left(v_{k}, \phi_{k}\right)-h_{k}\right\|_{2, \hat{\Omega}_{i, j}^{k}}^{2}\right. \\
& \left.+\sum_{l=1}^{L}\left\|u_{l}^{p+1}(\xi, \eta)\right\|_{2, S}^{2}\right) .
\end{aligned}
$$

Let

$$
\begin{aligned}
& y_{i, j}^{k}\left(v_{k}, \phi_{k}\right)=u_{i, j}^{k}\left(v_{k}, \phi_{k}\right)+r_{i, j}^{k}\left(v_{k}, \phi_{k}\right) \text { and } \\
& y_{l}^{p+1}(\xi, \eta)=u_{l}^{p+1}(\xi, \eta)+r_{l}^{p+1}(\xi, \eta) .
\end{aligned}
$$

Now we define a correction $\left\{\left\{s_{i, j}^{k}\left(v_{k}, \phi_{k}\right)\right\}_{i, j, k},\left\{s_{l}^{p+1}(\xi, \eta)\right\}_{l}\right\}$ such that $s_{i, 1}^{k}=0$ for all $i$ and $k, s_{i, j}^{k} \in H^{2}\left(\hat{\Omega}_{i, j}^{k}\right)$ for $2 \leq j \leq M$ and all $i$ and $k, s_{l}^{p+1} \in H^{2}(S)$ for $l=1, \ldots, L$ and $w=y+s \in H_{\beta}^{2,2}(\Omega)$.

Consider $\hat{\Omega}_{i, j}^{k}$ with $2 \leq j<M$. Let

$$
\begin{aligned}
& F_{1}\left(\phi_{k}\right)=-\left.\frac{1}{2}\left(y_{i, j}^{k}-y_{i, j+1}^{k}\right)\right|_{\hat{\gamma}_{1}}, \\
& G_{1}\left(\phi_{k}\right)=-\left.\frac{1}{2}\left(y_{i, j}^{k}-y_{i, j+1}^{k}\right) v_{v_{k}}\right|_{\hat{\gamma}_{1}}, \text { and } \\
& H_{1}\left(\phi_{k}\right)=-\left.\frac{1}{2}\left(y_{i, j}^{k}-y_{i, j+1}^{k}\right)_{\phi_{k}}\right|_{\hat{\gamma}_{1}} .
\end{aligned}
$$

In the same way we define $F_{l}, G_{l}$ and $H_{l}$ for $l=1, \ldots, 4$. If $\gamma_{l} \subseteq \partial \Omega$ for some $l, F_{l}, G_{l}$ and $H_{l}$ are defined to be identically zero on $\hat{\gamma}_{l}$. Now $F_{l}, G_{l}$ and $H_{l}$ are polynomials of degree $W$ that vanish at the end points $\hat{Q}_{l}$ and $\hat{Q}_{l+1}$ of $\hat{\gamma}_{l}$. If $\gamma_{3} \subseteq \partial \Omega_{i, 1}^{k} \cap \partial \Omega_{i, 2}^{k}$ for some $i, k$ then the factor of $\frac{1}{2}$ will be missing in the definition of $F_{3}\left(\phi_{k}\right), G_{3}\left(\phi_{k}\right)$ and $H_{3}\left(\phi_{k}\right)$. We wish to define $s_{i, j}^{k}\left(v_{k}, \phi_{k}\right)$ on $\hat{\Omega}_{i, j}^{k}$ such that $\left.s_{i, j}^{k}\right|_{\hat{\gamma}_{l}}=F_{l},\left.\left(s_{i, j}^{k}\right)_{v_{k}}\right|_{\hat{\gamma}_{l}}=G_{l}$ and $\left.\left(s_{i, j}^{k}\right)_{\phi_{k}}\right|_{\hat{\gamma}_{l}}=H_{l}$ for $l=1, \ldots, 4$.

We now cite Theorem 1.5.2.4 of [8]. The mapping $u \rightarrow\left\{\left\{f_{k}\right\}_{k=0}^{m-1},\left\{g_{k}\right\}_{k=0}^{m-1}\right\}$ defined by $f_{k}=\left.D_{\xi}^{k} u\right|_{\xi=0}, g_{l}=\left.D_{\eta}^{l} u\right|_{\eta=0}$ for $u \in D\left(\overline{\mathbb{R}_{+} \times \mathbb{R}_{+}}\right)$has a unique continuous extension as

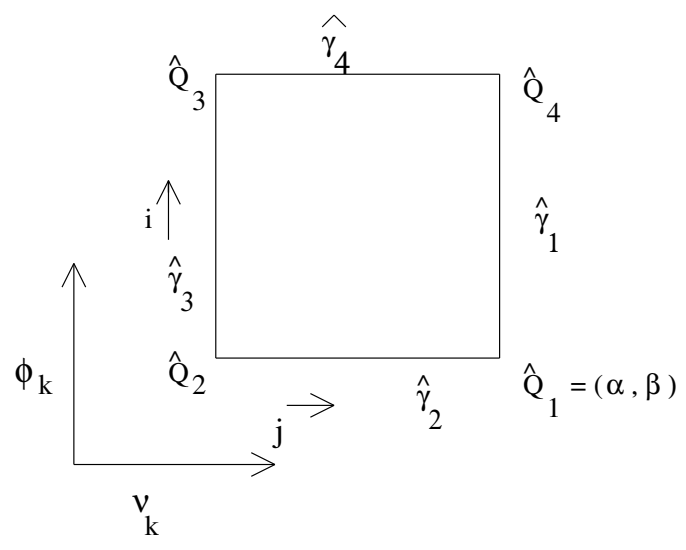


an operator from $W_{p}^{m}\left(\overline{\mathbb{R}_{+} \times \mathbb{R}_{+}}\right)$onto the subspace of

$$
T=\prod_{k=0}^{m-1} W^{m-k-1 / p}\left(\mathbb{R}_{+}\right) \times \prod_{l=0}^{m-1} W^{m-l-1 / p}\left(\mathbb{R}_{+}\right)
$$

defined by

(a) $D_{\eta}^{l} f_{k}(0)=D_{\xi}^{k} g_{l}(0), l+k<m-2 / p$ for all $p \neq 2$, and

(b) $\int_{0}^{\delta}\left|D_{\eta}^{l} f_{k}(t)-D_{\xi}^{k} g_{l}(t)\right|^{2} \mathrm{~d} t / t<\infty, l+k=m-1$ for $p=2$.

Hence using a partition of unity argument it is enough to show that

(i) $\int_{0}^{\delta}\left|D_{\phi_{k}} F_{1}(t+\beta)-H_{2}(\alpha-t)\right|^{2} \mathrm{~d} t / t$, and

(ii) $\int_{0}^{\delta}\left|G_{1}(t+\beta)-D_{v_{k}} F_{2}(\alpha-t)\right|^{2} \mathrm{~d} t / t$, are finite.

Conditions (i) and (ii) follow by applying the above theorem to a neighbourhood of the vertex $\hat{Q}_{1}=(\boldsymbol{\alpha}, \boldsymbol{\beta})$ of $\hat{\Omega}_{i, j}^{k}$.

Now

$$
\begin{aligned}
& \int_{0}^{\delta}\left|D_{\phi_{k}} F_{1}(t+\beta)-H_{2}(\alpha-t)\right|^{2} \mathrm{~d} t / t \\
& \quad \leq 2 \int_{0}^{\delta}\left|D_{\phi_{k}} F_{1}(t+\beta)\right|^{2} \mathrm{~d} t / t+2 \int_{0}^{\delta}\left|H_{2}(\alpha-t)\right|^{2} \mathrm{~d} t / t .
\end{aligned}
$$

Moreover from Theorem 4.82 in [12] we have that if $q(y)$ is a polynomial of degree $W$ on $I=(-1,1)$ such that $q(-1)=q(1)=0$, then

$$
\int_{-1}^{1} \frac{q^{2}(y)}{1-y^{2}} \mathrm{~d} y \leq C \ln W\|q\|_{L^{\infty}(I)}^{2} .
$$

Now by (7.4),

$$
\|q\|_{L^{\infty}(I)}^{2} \leq K \ln W\|q\|_{H^{1 / 2(I)}}^{2} .
$$

Hence we conclude that

$$
\int_{0}^{\delta}\left|D_{\phi_{k}} F_{1}(t+\beta)-H_{2}(\alpha-t)\right|^{2} \mathrm{~d} t / t \leq C(\ln W)^{2}\left(\left\|D_{\phi_{k}} F_{1}\right\|_{1 / 2, \hat{\gamma}_{1}}^{2}+\left\|H_{2}\right\|_{1 / 2, \hat{\gamma}_{1}}^{2}\right) .
$$

A similar result holds for (ii).

Hence we can define $\left\{\left\{s_{i, j}^{k}\left(v_{k}, \phi_{k}\right)\right\}_{i, j, k},\left\{s_{l}^{p+1}(\xi, \eta)\right\}_{l}\right\}$ such that $s_{i, 1}^{k}=0$ for all $i$ and $k$, $s_{i, j}^{k} \in H^{2}\left(\hat{\Omega}_{i, j}^{k}\right)$ for $j \geq 2, s_{l}^{p+1} \in H^{2}(S)$ and $w=y+s \in H_{\beta}^{2,2}(\Omega)$.

Let $v_{i, j}^{k}\left(v_{k}, \phi_{k}\right)=r_{i, j}^{k}\left(v_{k}, \phi_{k}\right)+s_{i, j}^{k}\left(v_{k}, \phi_{k}\right)$ and $v_{l}^{p+1}(\xi, \eta)=r_{l}^{p+1}(\xi, \eta)+s_{l}^{p+1}(\xi, \eta)$. 
Now from (7.7) we conclude that there is a constant $K$ such that

$$
\begin{aligned}
& \sum_{k=1}^{p} \sum_{i=1}^{I_{k}} \sum_{j=2}^{M}\left(\rho \mu_{k}^{M+1-j}\right)^{-2 \lambda_{k}}\left\|s_{i, j}^{k}\left(v_{k}, \phi_{k}\right)\right\|_{2, \hat{\Omega}_{i, j}^{k}}^{2}+\sum_{l=1}^{L}\left\|s_{l}^{p+1}(\xi, \eta)\right\|_{2, S}^{2} \\
& \leq K(\ln W)^{2}\left(\sum_{k=1}^{p} \sum_{\gamma_{s} \subseteq \Omega^{k} \cup B_{\rho}^{k}, \mu\left(\hat{\gamma}_{s}\right)<\infty} d\left(A_{k}, \gamma_{s}\right)^{-2 \lambda_{k}}\right. \\
& \times\left(\left\|\left[u^{k}\right]\right\|_{0, \gamma_{s}}^{2}+\left\|\left[\left(u_{v_{k}}^{k}\right)^{a}\right]\right\|_{1 / 2, \gamma_{s}}^{2}+\left\|\left[\left(u_{\phi_{k}}^{k}\right)^{a}\right]\right\|_{1 / 2, \hat{\gamma}_{s}}^{2}\right) \\
&\left.+\sum_{\gamma_{s} \subseteq \Omega^{p+1}}\left(\left\|\left[u^{p+1}\right]\right\|_{0, \gamma_{s}}^{2}+\left\|\left[\left(u_{x_{1}}^{p+1}\right)^{a}\right]\right\|_{1 / 2, \gamma_{s}}^{2}+\left\|\left[\left(u_{x_{2}}^{p+1}\right)^{a}\right]\right\|_{1 / 2, \gamma_{s}}^{2}\right)\right) \\
&+\varepsilon_{W}\left(\sum_{k=1}^{p} \sum_{i=1}^{I_{k}} \sum_{j=2}^{M}\left(\rho \mu_{k}^{M+1-j}\right)^{-2 \lambda_{k}}\left\|u_{i, j}^{k}\left(v_{k}, \phi_{k}\right)-h_{k}\right\|_{2, \hat{\Omega}_{i, j}^{k}}^{2}\right. \\
&\left.+\sum_{l=1}^{L}\left\|u_{l}^{p+1}(\xi, \eta)\right\|_{2, S}^{2}\right) .
\end{aligned}
$$

Combining (7.5) and (7.8) gives the estimate (7.1).

We now prove the last result of this section.

Lemma 7.2. Let $w=u+v \in H_{\beta}^{2,2}(\Omega)$. Here $\left\{\left\{u_{i, j}^{k}\left(v_{k}, \phi_{k}\right)\right\}_{i, j, k},\left\{u_{l}^{p+1}(\xi, \eta)\right\}_{l}\right\} \in \Pi^{M, W}$ and $\left\{\left\{v_{i, j}^{k}\left(v_{k}, \phi_{k}\right)\right\}_{i, j, k},\left\{v_{l}^{p+1}(\xi, \eta)\right\}_{l}\right\}$ is as defined in Lemma 7.1. Then the estimate

$$
\begin{aligned}
& \|w\|_{H_{\beta}^{\frac{3}{2}, \frac{3}{2}}\left(\Gamma^{[0]}\right)}^{2}+\left\|\left(\frac{\partial w}{\partial N}\right)_{A}\right\|_{H_{\beta}^{\frac{1}{2}, \frac{1}{2}}\left(\Gamma^{[1]}\right)}^{2} \\
& \leq C(\ln W)^{2}\left(\sum_{k: \partial \Omega^{k} \cap \Gamma^{[0] \neq \emptyset}}^{p}\left|h_{k}\right|^{2}+\sum_{l \in \mathscr{D}} \sum_{k=l-1}^{l} \sum_{\gamma_{s} \subseteq \partial \Omega^{k} \cap \Gamma_{l}, \mu\left(\hat{\gamma}_{s}\right)<\infty}\right.
\end{aligned}
$$

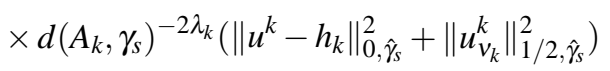

$$
\begin{aligned}
& +\sum_{l \in \mathscr{N}} \sum_{k=l-1}^{l} \sum_{\gamma_{s} \subseteq \partial \Omega^{k} \cap \Gamma_{l}, \mu\left(\hat{\gamma}_{s}\right)<\infty} d\left(A_{k}, \gamma_{s}\right)^{-2 \lambda_{k}}\left\|\left(\frac{\partial u^{k}}{\partial n}\right)_{\tilde{A}^{k}}^{a}\right\|_{1 / 2, \hat{\gamma}_{s}}^{2} \\
& +\sum_{l \in \mathscr{D}} \sum_{\gamma_{s} \subseteq \partial \Omega^{p+1} \cap \Gamma_{l}}\left(\left\|u^{p+1}\right\|_{0, \gamma_{s}}^{2}+\left\|\left(\frac{\partial u^{p+1}}{\partial T}\right)^{a}\right\|_{1 / 2, \gamma_{s}}^{2}\right) \\
& +\sum_{l \in \mathscr{N}} \sum_{\gamma_{s} \subseteq \partial \Omega^{p+1} \cap \Gamma_{l}}\left\|\left(\frac{\partial u^{p+1}}{\partial N}\right)_{A}^{a}\right\|_{1 / 2, \gamma_{s}}^{2}+\sum_{k=1}^{p} \sum_{\gamma_{s} \subseteq \Omega^{k} \cup B_{\rho}^{k}, \mu\left(\hat{\gamma}_{s}\right)<\infty}
\end{aligned}
$$




$$
\begin{aligned}
& \times d\left(A_{k}, \gamma_{s}\right)^{-2 \lambda_{k}}\left(\left\|\left[u^{k}\right]\right\|_{0, \gamma_{s}}^{2}+\left\|\left[\left(u_{v_{k}}^{k}\right)^{a}\right]\right\|_{1 / 2, \gamma_{s}}^{2}+\left\|\left[\left(u_{\phi_{k}}^{k}\right)^{a}\right]\right\|_{1 / 2, \gamma_{s}}^{2}\right) \\
& \left.+\sum_{\gamma_{s} \subseteq \Omega^{p+1}}\left(\left\|\left[u^{p+1}\right]\right\|_{0, \gamma_{s}}^{2}+\left\|\left[\left(u_{x_{1}}^{p+1}\right)^{a}\right]\right\|_{1 / 2, \gamma_{s}}^{2}+\left\|\left[\left(u_{x_{2}}^{p+1}\right)^{a}\right]\right\|_{1 / 2, \gamma_{s}}^{2}\right)\right) \\
& +\varepsilon_{W}\left(\sum_{k=1}^{p}\left|h_{k}\right|^{2}+\sum_{k=1}^{p} \sum_{i=1}^{I_{k}} \sum_{j=2}^{M}\left(\rho \mu_{k}^{M+1-j}\right)^{-2 \lambda_{k}}\left\|u_{i, j}^{k}\left(v_{k}, \phi_{k}\right)-h_{k}\right\|_{2, \hat{\Omega}_{i, j}^{k}}^{2}\right. \\
& \left.+\sum_{l=1}^{L}\left\|u_{l}^{p+1}(\xi, \eta)\right\|_{2, S}^{2}\right)
\end{aligned}
$$

holds. Here $\varepsilon_{W}$ is exponentially small in W. Now

$$
\|w\|_{H_{\beta}^{\frac{3}{2}, \frac{3}{2}}\left(\Gamma^{[0]}\right)}=\inf _{\left.q\right|_{\Gamma[0]}=w}\left\{\|q\|_{H_{\beta}^{2,2}(\Omega)}\right\} .
$$

Let $\theta_{k} \in C^{2}(\mathbb{R})$ such that $\theta_{k}=1$ for $r_{k} \leq \rho \mu_{k}$ and $\theta_{k}=0$ for $r_{k} \geq \rho$. Let $q_{k}=q \theta_{k}$ and $q_{0}=1-\sum_{k=1}^{p} q_{k}$. Let $\theta_{0}=1-\sum_{k=1}^{p} \theta_{k}$. Define $\Omega_{\rho \mu_{k}}^{k}=\left\{x: d\left(A_{k}, x\right)<\rho \mu_{k}\right\}$ for $k=1, \ldots, p$ and let $\tilde{\Omega}^{p+1}=\Omega \backslash \bigcup_{k=1}^{p} \bar{\Omega}_{\rho \mu_{k}}^{k}$. Then it can be concluded that

$$
\begin{aligned}
& \|w\|_{H_{\beta}^{\frac{3}{2}}, \frac{3}{2}}^{2}\left(\Gamma^{[0]}\right) \\
& \leq C\left(\sum_{k:\left.\partial \Omega^{k} \cap \Gamma^{[0] \neq \emptyset} q_{k}\right|_{\partial \Omega^{k} \cap \Gamma^{[0]}}=w \theta_{k}}\left\{\left\|q_{k}\right\|_{H_{\beta}^{2,2}\left(\Omega^{k}\right)}^{2}\right\}+\inf _{\left.q_{0}\right|_{\partial \tilde{\Omega}^{p+1} \cap \Gamma^{[0]}}=w \theta_{0}}\left\{\left\|q_{0}\right\|_{H^{2}\left(\tilde{\Omega}^{p+1}\right)}^{2}\right\}\right) .
\end{aligned}
$$

Now using (3.9) we have

$$
\left\|q_{k}\right\|_{H_{\beta}^{2,2}\left(\Omega^{k}\right)}^{2} \leq C\left(\left|h_{k}\right|^{2}+\left\|\left(q_{k}\left(v_{k}, \phi_{k}\right)-h_{k}\right) \mathrm{e}^{-2\left(1-\beta_{k}\right) v_{k}}\right\|_{2, \hat{\Omega}^{k}}^{2}\right) .
$$

Let us choose the cut-off function $\theta_{k}$ to be a piecewise polynomial such that

$$
\begin{aligned}
\theta_{k}\left(v_{k}\right) & =1 \quad \text { for } v_{k} \leq \ln \left(\rho \mu_{k}\right), \\
\theta_{k}\left(\ln \left(\rho \mu_{k}\right)\right) & =1, \theta_{k}^{(1)}\left(\ln \left(\rho \mu_{k}\right)\right)=0, \theta_{k}^{(2)}\left(\ln \left(\rho \mu_{k}\right)\right)=0, \\
\theta_{k}(\ln \rho) & =0, \theta_{k}^{(1)}(\ln \rho)=0, \theta_{k}^{(2)}(\ln \rho)=0, \quad \text { and } \\
\theta_{k}\left(v_{k}\right) & =0 \quad \text { for } v_{k} \geq \ln \rho .
\end{aligned}
$$

Here $\theta_{k}^{(l)}$ denotes the $l$ th derivative of $\theta_{k}$ with respect to $v_{k}$. Then $\theta_{k}$ is a polynomial of degree five in $v_{k}$ for $\ln \left(\rho \mu_{k}\right) \leq v_{k} \leq \ln \rho$. Now using (7.10) and (7.11) we have 


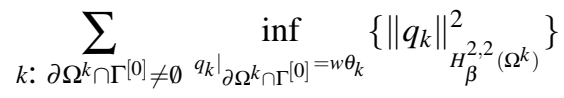

$$
\begin{aligned}
& \leq C\left(\sum_{k: \partial \Omega^{k} \cap \Gamma^{[0]}}\left|h_{k}\right|^{2}+\sum_{k: \Gamma_{k} \cap \Gamma^{[0]} \neq \emptyset}\left\|\left(q_{k}\left(v_{k}, \psi_{l}^{k}\right)-h_{k}\right) \mathrm{e}^{-2\left(1-\beta_{k}\right) v_{k}}\right\|_{3 / 2,(-\infty, l n \rho)}^{2}\right) \\
& \left.+\sum_{k: \Gamma_{k+1} \cap \Gamma^{[0]} \neq \emptyset}\left\|\left(q_{k}\left(v_{k}, \psi_{u}^{k}\right)-h_{k}\right) \mathrm{e}^{-2\left(1-\beta_{k}\right) v_{k}}\right\|_{3 / 2,(-\infty, l n \rho)}^{2}\right) .
\end{aligned}
$$

Let $\eta_{j}^{k}=\ln \rho+(M+1-j) \ln \mu_{k}$ and $I_{j}^{k}=\left(\eta_{j-1}^{k}, \eta_{j}^{k}\right)$. Then

$$
\begin{aligned}
& \left\|\left(q_{k}\left(v_{k}, \psi_{u}^{k}\right)-h_{k}\right) \mathrm{e}^{-2\left(1-\beta_{k}\right) v_{k}}\right\|_{3 / 2,(-\infty, l n \rho)}^{2} \\
& \leq C\left\{\sum_{j=2}^{M+1}\left\|\left(q_{k}\left(v_{k}, \psi_{u}^{k}\right)-h_{k}\right) \mathrm{e}^{-2\left(1-\beta_{k}\right) v_{k}}\right\|_{0,\left(\eta_{j-1}^{k}, \eta_{j}^{k}\right)}^{2}\right. \\
& \quad+\sum_{j=2}^{M+1}\left\|\left(\left(q_{k}\left(v_{k}, \psi_{u}^{k}\right)-h_{k}\right) \mathrm{e}^{-2\left(1-\beta_{k}\right) v_{k}}\right) v_{k}\right\|_{1 / 2,\left(\eta_{j-1}^{k}, \eta_{j}^{k}\right)}^{2} \\
& \quad+\sum_{j=2}^{M+1} \int_{0}^{\delta} \mid \frac{\mathrm{d}}{\mathrm{d} s}\left(\left(q_{k}\left(s, \psi_{u}^{k}\right)-h_{k}\right) \mathrm{e}^{-\left(1-\beta_{k}\right) s}\right)\left(\eta_{j}^{k}+\sigma\right) \\
& \left.\quad-\left.\frac{\mathrm{d}}{\mathrm{d} s}\left(\left(q_{k}\left(s, \psi_{u}^{k}\right)-h_{k}\right) \mathrm{e}^{-\left(1-\beta_{k}\right) s}\right)\left(\eta_{j}^{k}-\sigma\right)\right|^{2} \frac{\mathrm{d} \sigma}{\sigma}\right\} .
\end{aligned}
$$

Here $\delta>0$. Now

$$
\begin{aligned}
& \int_{0}^{\delta} \mid \frac{\mathrm{d}}{\mathrm{d} s}\left(\left(q_{k}\left(s, \psi_{u}^{k}\right)-h_{k}\right) \mathrm{e}^{-\left(1-\beta_{k}\right) s}\right)\left(\eta_{j}^{k}+\sigma\right) \\
& \quad-\left.\frac{\mathrm{d}}{\mathrm{d} s}\left(\left(q_{k}\left(s, \psi_{u}^{k}\right)-h_{k}\right) \mathrm{e}^{-\left(1-\beta_{k}\right) s}\right)\left(\eta_{j}^{k}-\sigma\right)\right|^{2} \frac{\mathrm{d} \sigma}{\sigma} \\
& \leq K\left(\sum_{l=0}^{1} \int_{0}^{\delta} \mid \frac{\mathrm{d}^{l}}{\mathrm{~d} s^{l}}\left(q_{k}-h_{k}\right)\left(\eta_{j}^{k}+\sigma, \psi_{u}^{k}\right)\right. \\
&-\left.\frac{\mathrm{d}^{l}}{\mathrm{~d} s^{l}}\left(q_{k}-h_{k}\right)\left(\eta_{j}^{k}-\sigma, \psi_{u}^{k}\right)\right|^{2} \mathrm{e}^{-2\left(1-\beta_{k}\right)\left(\eta_{j}^{k}+\sigma\right)} \frac{\mathrm{d} \sigma}{\sigma} \\
&+\sum_{l=0}^{1} \int_{0}^{\delta}\left|\frac{\mathrm{d}^{l}}{\mathrm{~d} s^{l}}\left(q_{k}-h_{k}\right)\left(\eta_{j}^{k}-\sigma, \psi_{u}^{k}\right)\right|^{2} \\
&\left.\times\left(\mathrm{e}^{-2\left(1-\beta_{k}\right)\left(\eta_{j}^{k}+\sigma\right)}-\mathrm{e}^{-2\left(1-\beta_{k}\right)\left(\eta_{j}^{k}-\sigma\right)}\right) \frac{\mathrm{d} \sigma}{\sigma}\right) .
\end{aligned}
$$


Hence

$$
\begin{aligned}
& \left\|\left(q_{k}\left(v_{k}, \psi_{u}^{k}\right)-h_{k}\right) \mathrm{e}^{-2\left(1-\beta_{k}\right) v_{k}}\right\|_{3 / 2,(-\infty, \ln \rho)}^{2} \\
& \leq C\left(\sum_{\gamma_{s} \subseteq \Gamma^{[0]} \cap \Gamma_{k+1}, \mu\left(\hat{\gamma}_{s}\right)<\infty} d\left(A_{k}, \gamma_{s}\right)^{-2 \lambda_{k}}\right. \\
& \left.\quad \times\left(\left\|\left(q_{k}-h_{k}\right)\right\|_{0, \gamma_{s}}^{2}+\left\|\frac{\mathrm{d} q_{k}}{\mathrm{~d} v_{k}}\right\|_{1 / 2, \hat{\gamma}_{s}}^{2}+\ln W\left\|\left(q_{k}-h_{k}\right)\right\|_{1, \infty, \hat{\gamma}_{s}}^{2}\right)\right) .
\end{aligned}
$$

This follows from Theorem 4.82 in [12] which states that if $p(y)$ is a polynomial of degree $N$ in $y$ such that $p(1)=p(-1)=0$, then

$$
\int_{-1}^{1} \frac{p^{2}(y)}{1-y^{2}} \mathrm{~d} y \leq C \ln N\|p\|_{L^{\infty}(\bar{I})}^{2}
$$

Now $q_{k}\left(v_{k}, \psi_{u}^{k}\right)-h_{k}=\theta_{k} w\left(v_{k}, \psi_{u}^{k}\right)-h_{k}=\theta_{k}\left(w\left(v_{k}, \psi_{u}^{k}\right)-h_{k}\right)+\left(\theta_{k}-1\right) h_{k}$.

Moreover $w=r+s+u$, as has been defined in Lemma 7.1. Here $w\left(v_{k}, \psi_{u}^{k}\right)$ is a polynomial of degree $W, s\left(v_{k}, \psi_{u}^{k}\right)=0$ and $r\left(v_{k}, \psi_{u}^{k}\right)$ is a polynomial degree four.

Hence using (7.4) and (7.13) we conclude that

$$
\begin{aligned}
& \left\|\left(q_{k}\left(v_{k}, \psi_{u}^{k}\right)-h_{k}\right) \mathrm{e}^{-2\left(1-\beta_{k}\right) v_{k}}\right\|_{3 / 2,(-\infty, \ln \rho)}^{2} \\
& \quad \leq C\left(\sum _ { \gamma _ { s } \subseteq \Gamma _ { k + 1 } \cap \partial \Omega ^ { k } } d ( A _ { k } , \gamma _ { s } ) ^ { - 2 \lambda _ { k } } \left(( \operatorname { l n } W ) ^ { 2 } \left(\left\|\left(u\left(v_{k}, \psi_{u}^{k}\right)-h_{k}\right)\right\|_{3 / 2, \gamma_{s}}^{2}\right.\right.\right. \\
& \left.\left.\left.\quad+\left|h_{k}\right|^{2}\right)+\ln W\left\|r\left(v_{k}, \psi_{u}^{k}\right)\right\|_{3 / 2, \gamma_{s}}^{2}\right)\right) .
\end{aligned}
$$

Hence using (7.5) and (7.14) it can be concluded that

$$
\begin{aligned}
& \sum_{k: \partial \Omega^{k} \cap \Gamma^{[0] \neq \emptyset}} \inf _{\left.q_{k}\right|_{\partial \Omega^{k} \cap[0]=w \theta_{k}}}\left\{\left\|q_{k}\right\|_{H_{\beta}^{2,2}\left(\Omega^{k}\right)}^{2}\right\} \\
& \leq C(\ln W)^{2}\left(\sum_{k=1}^{p}\left|h_{k}\right|^{2}+\sum_{l \in \mathscr{D}} \sum_{k=l-1}^{l} \sum_{\gamma_{s} \subseteq \partial \Omega^{k} \cap \Gamma_{l}, \mu\left(\hat{\gamma}_{s}\right)<\infty} d\left(A_{k}, \gamma_{s}\right)^{-2 \lambda_{k}}\right. \\
& \quad \times\left(\left\|u^{k}-h_{k}\right\|_{0, \gamma_{s}}^{2}+\left\|u_{v_{k}}^{k}\right\|_{1 / 2, \hat{\gamma}_{s}}^{2}\right)+\sum_{k=1}^{p} \sum_{\gamma_{s} \subseteq \Omega^{k} \cup B_{\rho}^{k}, \mu\left(\hat{\gamma}_{s}\right)<\infty} d\left(A_{k}, \gamma_{s}\right)^{-2 \lambda_{k}} \\
& \quad \times\left(\left\|\left[u^{k}\right]\right\|_{0, \hat{\gamma}_{s}}^{2}+\left\|\left[\left(u_{v_{k}}^{k}\right)^{a}\right]\right\|_{1 / 2, \hat{\gamma}_{s}}^{2}+\left\|\left[\left(u_{\phi_{k}}^{k}\right)^{a}\right]\right\|_{1 / 2, \gamma_{s}}^{2}\right) \\
& \left.\quad+\sum_{\gamma_{s} \subseteq \Omega^{p+1}}\left(\left\|\left[u^{p+1}\right]\right\|_{0, \gamma_{s}}^{2}+\left\|\left[\left(u_{x_{1}}^{p+1}\right)^{a}\right]\right\|_{1 / 2, \gamma_{s}}^{2}+\left\|\left[\left(u_{x_{2}}^{p+1}\right)^{a}\right]\right\|_{1 / 2, \gamma_{s}}^{2}\right)\right)
\end{aligned}
$$




$$
\begin{aligned}
& +\varepsilon_{W}\left(\sum_{k=1}^{p} \sum_{j=2}^{M} \sum_{i=1}^{I_{k}}\left(\rho \mu_{k}^{M+1-j}\right)^{-2 \lambda_{k}}\left\|u_{i, j}^{k}\left(v_{k}, \phi_{k}\right)-h_{k}\right\|_{2, \hat{S}_{i, j}^{k}}^{2}\right. \\
& \left.+\sum_{k=1}^{p}\left|h_{k}\right|^{2}+\sum_{l=1}^{L}\left\|u_{l}^{p+1}(\xi, \eta)\right\|_{2, S}^{2}\right) .
\end{aligned}
$$

In the same way we can show that

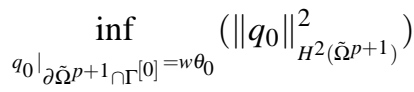

$$
\begin{aligned}
& \leq C(\ln W)^{2}\left(\sum_{k: \partial \Omega^{k} \cap \Gamma^{[0] \neq \emptyset}}^{p}\left|h_{k}\right|^{2}+\sum_{l \in \mathscr{D}} \sum_{k=l-1}^{l} \sum_{\gamma_{s} \subseteq \partial \Omega^{k} \cap \Gamma_{l}, \mu\left(\hat{\gamma}_{s}\right)<\infty}\right. \\
& \times d\left(A_{k}, \gamma_{s}\right)^{-2 \lambda_{k}}\left(\left\|u^{k}-h_{k}\right\|_{0, \hat{\gamma}_{s}}^{2}+\left\|u_{v_{k}}^{k}\right\|_{1 / 2, \hat{\gamma}_{s}}^{2}\right)+\sum_{k=1}^{p} \sum_{\gamma_{s} \subseteq \Omega^{k} \cup B_{\rho}^{k}, \mu\left(\hat{\gamma}_{s}\right)<\infty} \\
& \times d\left(A_{k}, \gamma_{s}\right)^{-2 \lambda_{k}}\left(\left\|\left[u^{k}\right]\right\|_{0, \hat{\gamma}_{s}}^{2}+\left\|\left[\left(u_{v_{k}}^{k}\right)^{a}\right]\right\|_{1 / 2, \hat{\gamma}_{s}}^{2}+\left\|\left[\left(u_{\phi_{k}}^{k}\right)^{a}\right]\right\|_{1 / 2, \hat{\gamma}_{s}}^{2}\right) \\
& +\sum_{\gamma_{s} \subseteq \Omega^{p+1}}\left(\left\|\left[u^{p+1}\right]\right\|_{0, \gamma_{s}}^{2}+\left\|\left[\left(u_{x_{1}}^{p+1}\right)^{a}\right]\right\|_{1 / 2, \gamma_{s}}^{2}+\left\|\left[\left(u_{x_{2}}^{p+1}\right)^{a}\right]\right\|_{1 / 2, \gamma_{s}}^{2}\right) \\
& \left.+\sum_{l \in \mathscr{D}} \sum_{\gamma_{s} \subseteq \partial \Omega^{p+1} \cap \Gamma_{l}}\left(\left\|u^{p+1}\right\|_{0, \gamma_{s}}^{2}+\left\|\left(\frac{\partial u^{p+1}}{\partial T}\right)^{a}\right\|_{1 / 2, \gamma_{s}}^{2}\right)\right) \\
& +\varepsilon_{W}\left(\sum_{k=1}^{p} \sum_{j=2}^{M} \sum_{i=1}^{I_{k}}\left(\rho \mu_{k}^{M+1-j}\right)^{-2 \lambda_{k}}\left\|u_{i, j}^{k}\left(v_{k}, \phi_{k}\right)-h_{k}\right\|_{2, \hat{\Omega}_{i, j}^{k}}^{2}\right. \\
& \left.+\sum_{k=1}^{p}\left|h_{k}\right|^{2}+\sum_{l=1}^{L}\left\|u_{l}^{p+1}(\xi, \eta)\right\|_{2, S}^{2}\right)
\end{aligned}
$$

Now

$$
\left\|\left(\frac{\partial w}{\partial N}\right)_{A}\right\|_{H_{\beta}^{\frac{1}{2}, \frac{1}{2}\left(\Gamma^{[1]}\right)}}^{2}=\inf _{q_{\Gamma^{[1]}}=\left(\frac{\partial w}{\partial N}\right)_{A}}\left(\|q\|_{H_{\beta}^{1,1}(\Omega)}^{2}\right) .
$$

Here

$$
\|q\|_{H_{\beta}^{1,1}(\Omega)}^{2}=\|q\|_{L^{2}(\Omega)}^{2}+\sum_{|\alpha|=1}\left\|\Phi_{\beta} D^{\alpha} q\right\|_{L^{2}(\Omega)}^{2} .
$$


Let $\theta_{k} \in C^{2}(\mathbb{R})$ be as defined earlier and $q_{k}=q \theta_{k}$. Let $q_{0}=1-\sum_{k=1}^{p} q_{k}$. Then, as before

$$
\begin{aligned}
& \left\|\left(\frac{\partial w}{\partial N}\right)_{A}\right\|_{H_{\beta}^{\frac{1}{2}, \frac{1}{2}}\left(\Gamma^{[1]}\right)}^{2} \\
& \quad \leq C\left(\inf _{\left.q_{k}\right|_{\partial \Omega^{k} \cap \Gamma^{[1]}}=\left(\frac{\partial w}{\partial N}\right)_{A} \theta_{k}}\left(\left\|q_{k}\right\|_{H_{\beta}^{1,1}\left(\Omega^{k}\right)}^{2}\right)+\operatorname{qun}_{\left.q_{0}\right|_{\partial \tilde{\Omega}^{p+1} \cap \Gamma^{[1]}}=\left(\frac{\partial w}{\partial N}\right)_{A} \theta_{0}}\left(\left\|q_{0}\right\|_{H^{1}\left(\tilde{\Omega}^{p+1}\right)}^{2}\right)\right) .
\end{aligned}
$$

Now

$$
\int_{\hat{\Omega}^{k}} \mathrm{e}^{2 \beta_{k} v_{k}}\left|q\left(v_{k}, \phi_{k}\right)\right|^{2} \mathrm{~d} v_{k} \mathrm{~d} \phi_{k} \leq K\|q\|_{H_{\beta}^{1,1}\left(\Omega^{k}\right)}^{2}
$$

for $\beta_{k}>0$. And so

$$
\int_{\hat{\Omega}^{k}} \mathrm{e}^{2 \beta_{k} v_{k}}\left|q_{k}\left(v_{k}, \phi_{k}\right)\right|^{2} \mathrm{~d} v_{k} \mathrm{~d} \phi_{k} \leq K\left\|q_{k}\right\|_{H_{\beta}^{1,1}\left(\Omega^{k}\right)}^{2} .
$$

Hence for $0<\beta_{k}<1$, there exists a constant $C$ such that

$$
\begin{aligned}
& \frac{1}{C}\left(\sum_{k=1}^{p} \inf _{\left.q_{k}\right|_{\partial \hat{\Omega}^{k} \cap \Gamma^{[1]}}=\mathrm{e}^{-v_{k}}\left(\frac{\partial w}{\partial n}\right)_{\tilde{A}^{k}} \theta_{k}}\right. \\
& \left.\times\left(\int_{\hat{\Omega}^{k}} \mathrm{e}^{2 \beta_{k} v_{k}} \sum_{|\alpha| \leq 1}\left|D_{v_{k}, \phi_{k}}^{\alpha} q_{k}\left(v_{k}, \phi_{k}\right)\right|^{2} \mathrm{~d} v_{k} \mathrm{~d} \phi_{k}\right)\right) \\
& \leq \sum_{k=1}^{p} \inf _{\left.q_{k}\right|_{\partial \hat{\Omega}^{k} \cap \Gamma[1]} \mathrm{e}^{-v_{k}}\left(\frac{\partial w}{\partial n}\right)_{\tilde{A}^{k}} \theta_{k}}\left(\left\|q_{k}\right\|_{H_{\beta}^{1,1}\left(\Omega^{k}\right)}^{2}\right) \\
& \leq C\left(\sum_{k=1}^{p} \inf _{\left.q_{k}\right|_{\partial \hat{\Omega}^{k} \cap \Gamma^{[1]}}=\mathrm{e}^{-v_{k}}\left(\frac{\partial w}{\partial n}\right)_{\tilde{A}^{k}} \theta_{k}}\right. \\
& \left.\times\left(\int_{\hat{\Omega}^{k}} \mathrm{e}^{2 \beta_{k} v_{k}} \sum_{|\alpha| \leq 1}\left|D_{v_{k}, \phi_{k}}^{\alpha} q_{k}\left(v_{k}, \phi_{k}\right)\right|^{2} \mathrm{~d} v_{k} \mathrm{~d} \phi_{k}\right)\right) .
\end{aligned}
$$

Thus by similar arguments as before it can be shown that

$$
\begin{aligned}
& \left\|\left(\frac{\partial w}{\partial N}\right)_{A}\right\|^{2} \\
& H_{\beta}^{\frac{1}{2}, \frac{1}{2}}\left(\Gamma^{[1]}\right) \\
& \leq C(\ln W)^{2}\left(\sum_{l \in \mathscr{N}} \sum_{k=l-1}^{l} \sum_{\gamma_{s} \subseteq \partial \Omega^{k} \cap \Gamma_{l}, \mu\left(\hat{\gamma}_{s}\right)<\infty} d\left(A_{k}, \gamma_{s}\right)^{-2 \lambda_{k}}\right.
\end{aligned}
$$




$$
\begin{aligned}
& \times\left\|\left(\frac{\partial u^{k}}{\partial n}\right)_{\tilde{A}^{k}}^{a}\right\|_{1 / 2, \gamma_{s}}^{2}+\sum_{l \in \mathscr{N}} \sum_{\gamma_{s} \subseteq \partial \Omega^{p+1} \cap \Gamma_{l}}\left\|\left(\frac{\partial u^{p+1}}{\partial N}\right)_{A}^{a}\right\|_{1 / 2, \gamma_{s}}^{2} \\
& +\sum_{k=1}^{p} \sum_{\gamma_{s} \subseteq \Omega^{k} \cup B_{\rho}^{k}, \mu\left(\hat{\gamma}_{s}\right)<\infty} d\left(A_{k}, \gamma_{s}\right)^{-2 \lambda_{k}} \\
& \times\left(\left\|\left[u^{k}\right]\right\|_{0, \gamma_{s}}^{2}+\left\|\left[\left(u_{v_{k}}^{k}\right)^{a}\right]\right\|_{1 / 2, \gamma_{s}}^{2}+\left\|\left[\left(u_{\phi_{k}}^{k}\right)^{a}\right]\right\|_{1 / 2, \gamma_{s}}^{2}\right) \\
& \left.+\sum_{\gamma_{s} \subseteq \Omega^{p+1}}\left(\left\|\left[u^{p+1}\right]\right\|_{0, \gamma_{s}}^{2}+\left\|\left[\left(u_{x_{1}}^{p+1}\right)^{a}\right]\right\|_{1 / 2, \gamma_{s}}^{2}+\left\|\left[\left(u_{x_{2}}^{p+1}\right)^{a}\right]\right\|_{1 / 2, \gamma_{s}}^{2}\right)\right) \\
& +\varepsilon_{W}\left(\sum_{k=1}^{p} \sum_{j=2}^{M} \sum_{i=1}^{I_{k}}\left(\rho \mu_{k}^{M+1-j}\right)^{-2 \lambda_{k}}\left\|u_{i, j}^{k}\left(v_{k}, \phi_{k}\right)-h_{k}\right\|_{2, \hat{\Omega}_{i, j}^{k}}^{2}\right. \\
& \left.+\sum_{k=1}^{p}\left|h_{k}\right|^{2}+\sum_{l=1}^{L}\left\|u_{l}^{p+1}(\xi, \eta)\right\|_{2, S}^{2}\right)
\end{aligned}
$$

Combining (7.15)-(7.17) we obtain the required result.

\section{Acknowledgement}

This research is partly supported by CDAC (Center for Development of Advanced Computing, Pune).

\section{References}

[1] Babuska I, Craig A, Mandel J and Pitkaranta J, Efficient preconditioning for the p version of the finite element method in two dimensions, SIAM J. Num. Anal. 28 (1991) 624

[2] Babuska I and Guo B Q, Regularity of the solution of elliptic problems with piecewise analytic data, Part-I, SIAM J. Math. Anal. 19 (1988) 172-203

[3] Babuska I and Guo B Q, The h-p version of the finite element method on domains with curved boundaries, SIAM J. Num. Anal. 25 (1988) 837-861

[4] Babuska I and Guo B Q, Regularity of the solution of elliptic problems with piecewise analytic data, Part-II, SIAM J. Math. Anal. 20 (1989) 763-781

[5] Dutt P K and Bedekar S, Spectral methods for hyperbolic initial boundary value problems on parallel computers, J. Comput. Appl. Math. 134 (2001) 165-190

[6] Dutt P, Tomar S and Kumar R, Stability estimates for h-p spectral element methods for elliptic problems, Proc. Indian Acad. Sci (Math. Sci.) 112(4) (2002) 601-639

[7] Dutt P and Tomar S, Stability estimates for h-p spectral element methods for general elliptic problems on curvilinear domains, Proc. Indian Acad. Sci (Math. Sci.) 113 (2003) 395-429

[8] Grisvard P, Elliptic problems in non-smooth domains (Pitman Advanced Publishing Program) (1985)

[9] Guo B Q and Babuska I, On the regularity of elasticity problems with piecewise analytic data, Adv. Appl. Math. 14 (1993) 307-347 
[10] Guo B and Cao W, A preconditioner for the h-p version of the finite element method in two dimensions, Num. Math. 75 (1996) 59

[11] Karniadakis G and Spencer Sherwin J, Spectral/hp element methods for CFD, (Oxford University Press) (1999)

[12] Schwab Ch, p and h-p Finite element methods (Oxford: Clarendon Press) (1998)

[13] Tomar S K, Dutt P and Rathish Kumar B V, An efficient and exponentially accurate parallel h-p spectral element method for elliptic problems on polygonal domains-The Dirichlet case, Lecture Notes in Computer Science 2552, High Performance Computing HiPC (Springer-Verlag) (2002)

[14] Tomar S K, h-p Spectral element methods for elliptic problems on non-smooth domains using parallel computers, Ph.D. thesis (India: IIT Kanpur) (2001); Reprint available as Tec. Rep. no. 1631, Department of Applied Mathematics, University of Twente, The Netherlands. http://www.math.utwente.nl/publications 